\title{
Grasping visual illusions: Consistent data and no dissociation
}

\author{
Volker H. Franz and Karl R. Gegenfurtner \\ University of Giessen, Giessen, Germany
}

\begin{abstract}
The finding that the Ebbinghaus/Titchener illusion deceives perception but not grasping is usually seen as strong evidence for Goodale and Milner's (1992) notion of two parallel visual systems, one being conscious and deceived by the illusion (vision-for-perception) and the other being unconscious and not deceived (vision-for-action). However, this finding is controversial and led to studies with seemingly contradictory results. We argue that these results are not as contradictory as it might seem. Instead, studies consistently show similar effects of the illusion on grasping. The perceptual effects are strongly dependent on the specific perceptual measure employed. If, however, some methodological precautions are used, then these diverse perceptual results can be reconciled and point to a single internal size estimate that is used for perception and for grasping. This suggests that the Ebbinghaus illusion deceives a common representation of object size that is used by perception and action.
\end{abstract}

Keywords: Perception; Action; Dorsal; Ventral stream.

The question whether visual awareness (or "perception") is generated by similar processes and brain areas to those for visually guided motor behaviour is not settled. According to the perception-versus-action hypothesis of Goodale and Milner (1992; A. D. Milner \& Goodale, 1995), two different systems generate visual perception and visually guided actions. This notion was derived from a wide variety of arguments, most notably from studies on neuropsychological patients showing, for example, a double dissociation between grasping an object and perceiving its shape (Goodale et al., 1994; Goodale, Milner, Jakobson, \& Carey, 1991).
Later evidence from healthy humans seemed to strongly support this view. One very prominent line of evidence was the finding that contextual visual illusions, such as the Ebbinghaus/ Titchener illusion (Figure 1), affect perception to a much larger extent than grasping (Aglioti, DeSouza, \& Goodale, 1995). In the framework of the perception-versus-action hypothesis we could interpret this surprising finding in the following way: Milner and Goodale (1995) assume that the vision-for-perception system is based on allocentric coding. That is, an object is coded relative to neighbouring objects. The vision-for-action system on the other hand is assumed to code

Correspondence should be addressed to Volker Franz, Justus-Liebig-Universität Giessen, FB 06/Abt. Allgemeine Psychologie, Otto-Behaghel-Strasse 10F, 35394 Giessen, Germany (E-mail: volker.franz@psychol.uni-giessen.de).

We wish to thank David Rosenbaum for strong encouragement and Bruce Bridgeman for helpful comments on earlier versions of the manuscript. This work was supported by Grant DFG/FR 2100/1-2 and the research unit DFG/FOR 560 "Perception and Action" of the Deutsche Forschungsgemeinschaft (DFG). 
(a)

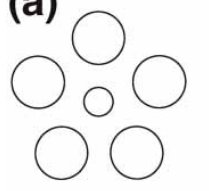

Large-Far

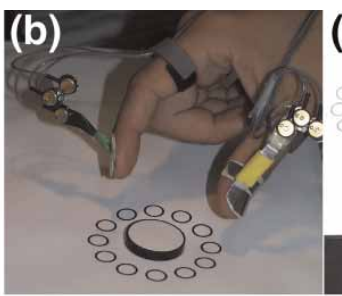

(c)

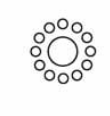

Small-Near

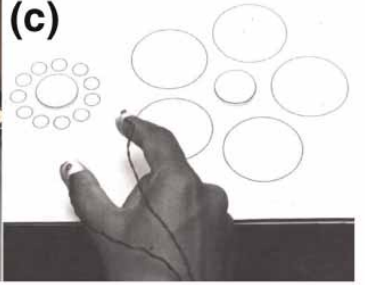

(d)

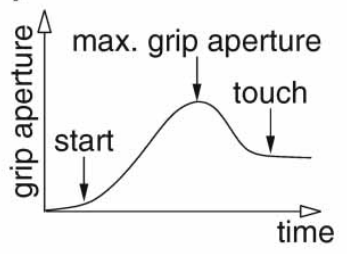

(e) Aglioti $95 \quad$ Haffenden 98 Haffenden $01 \quad$ Pavani $99 \quad$ Franz $00 \quad$ Franz 03

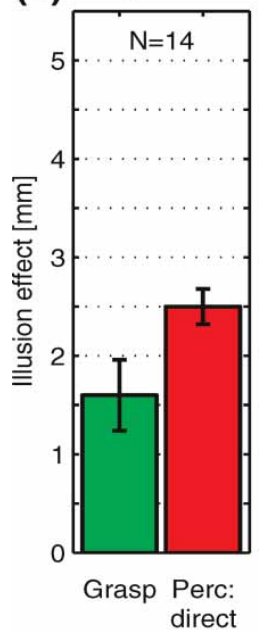

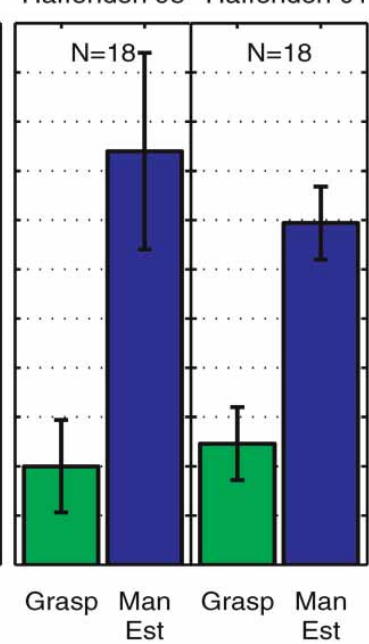

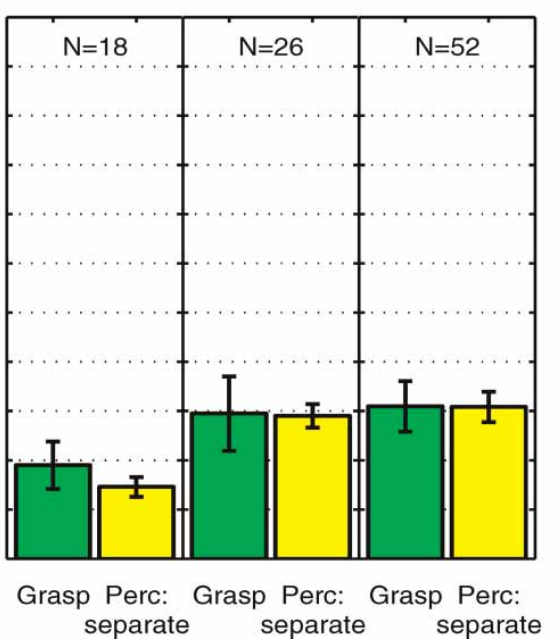

(f) Haffenden 01

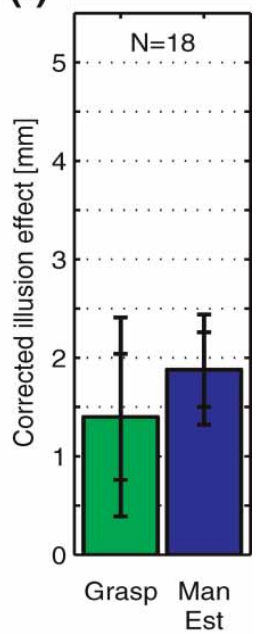

Figure 1. Ebbinghaus illusion and sample studies. (a) The Ebbinghaus (or Titchener) illusion (Ebbinghaus, 1902; Titchener, 1901; Wundt, 1898) as used in almost identical form in all example studies. The central circle is perceived as being smaller if surrounded by large context circles than if surrounded by small context circles. The illusion is strongest if the large context circles are relatively far from the central circle (large-far condition) and the small context circles are near (small-near condition). In all example studies, the central circle was replaced by a disc that could be grasped. (b) A participant grasping in the setup of Franz et al. (2000). (c) A participant grasping in the set-up of Aglioti et al. (1995). From "Size Contrast Illusions Deceive the Eye but Not the Hand", by S. Aglioti, J. F. X. DeSouza, E M. A. Goodale, 1995, Current Biology, 5, pp. 679-685. Copyright 1995 by Elsevier. Reprinted with permission. (d) When grasping, index finger and thumb open to a maximum grip aperture (MGA) before touching the target object. The MGA is linearly related to the size of the target object. (e) Illusion effects in the example studies. (f) Results of Haffenden et al. (2001) after correcting for the different slopes of the linear functions relating physical size to the dependent measure. Illusion effects are shown for the following dependent variables: "Grasp": MGA when grasping. "Perc: direct" and "Perc: separate": classic perceptual measures for direct and separate comparisons, respectively. "Man Est": manual estimation. Data are from: Aglioti et al. (1995, Fig. 5); Haffenden and Goodale (1998, personal communication, June 26, 1998); Haffenden et al. (2001, Fig. 1; conditions: "traditional small/large" and pp. 177-178); Pavani et al. (1999, p. 99, and personal communication, October 4, 1999); Franz et al. (2000), and Franz et al. (2003), own records. All error bars depict \pm 1 SEM. In (e) the SEMs were estimated using the delta method (i.e., a linear Taylor approximation, cf. Buonaccorsi, 2001, Franz, 2007). Because this method needs the correlation between dependent measure and slope (which were not reported), two error bars are depicted for $\rho=-.50$ (larger SEM) and $\rho=+.50$ (smaller SEM). The "true" SEM will probably be between these values. (Figure can be seen in colour online.)

objects in egocentric coordinates, relative to the effector used to interact with the object. Because the Ebbinghaus illusion is induced by the neighbouring context circles, it should only affect the allocentric coding in the vision-for-perception system. And because our conscious awareness is assumed to have access only to the vision-for- perception system, it cannot use the veridical size estimate in the action system, and therefore the hand should "know" sometimes the true, metrical size of objects better than does our conscious awareness. In short, "what we think we see may not always be what guides our actions" (Aglioti et al., 1995, p. 684). This surprising finding 
seemed to clearly corroborate the perceptionversus-action hypothesis suggested earlier by Goodale and Milner (1992).

However, this finding is highly controversial (Bruno, 2001; Carey, 2001; Franz, 2001; Franz, Gegenfurtner, Bülthoff, \& Fahle, 2000; Glover, 2002, 2004; D. Milner \& Dyde, 2003; Pavani, Boscagli, Benvenuti, Rabuffetti, \& Farnè, 1999; Smeets \& Brenner, 2001), such that one might get the impression that the data in this research area were simply messy. To the contrary, we argue that the data are surprisingly consistent and that they point to a simple, parsimonious explanation-namely, that both perception and action are deceived by contextual illusions in a similar way. This implies that we cannot count contextual illusions as evidence for the perception-versus-action hypothesis.

To make our argument, we concentrate on the Ebbinghaus illusion. The reason is that this illusion has been investigated most thoroughly and systematically and that it has "captured most of the limelight" (Carey, 2001, p. 109). The original interpretation that this illusion deceives conscious perception but not actions (Aglioti et al., 1995) has been for a long time the standard example in healthy observers for the dissociation between perception and action as proposed by Goodale and Milner (1992; Milner \& Goodale, 1995).

We show that quite simple, but sometimes subtle, methodological aspects can lead to the impression of a dissociation between perception and action that is, in our view, not warranted by the data. Of course, the question arises how our arguments can be generalized to other illusions and other actions. Given the huge literature on this topic, it is not possible to discuss all existing studies in detail. We do, however, discuss some of the most prominent issues in the second part of this article. Also we added an Appendix, discussing some more technical aspects that are relevant if one wanted to conduct a study on this topic.

The basic results and sources of controversy are shown in Figure 1e. The figure summarizes the results of six example studies that used similar illusion configurations, so that we can roughly compare the results. In the remainder of this article, we refer to these studies as the "example studies". Inspecting the figure, we can see two things: First, there are illusion effects on grasping, and these effects are quite similar across the different studies. This leads to our first question: If we assume that Goodale and Milner (1992; Milner \& Goodale, 1995) are correct, then why is there an illusion effect on grasping at all? Second, the different perceptual measures deviate substantially (compare the values for "Perc: direct", "Perc: separate", and "Man Est"). This leads to the second question: Which perceptual illusion should be compared to grasping? And why are the results of the perceptual measures so different? Before discussing these questions, we first need to give a little more technical detail on how the illusion effects are measured in this area of research.

\section{How were the illusion effects measured?}

Figure 1a shows the Ebbinghaus illusion as was used in almost identical form in all of the example studies. The central circle is perceived as being smaller if surrounded by large context circles than if surrounded by small context circles. The illusion is strongest (Girgus, Coren, \& Agdern, 1972) if the large context circles are relatively far from the central circle (large-far condition), and the small context circles are near (small-near condition). Further studies varied the distances of the context circles to investigate potential influences of obstacle avoidance mechanisms (Franz, Bülthoff, \& Fahle, 2003; Haffenden, Schiff, \& Goodale, 2001). These are discussed later.

To measure the effects of the Ebbinghaus illusion on grasping, researchers replaced the central circles of the Ebbinghaus figures with discs that could be grasped in a precision grip with index finger and thumb (Figure 1b, 1c). The grasping movements were recorded using optoelectronic systems (in most cases an Optotrak ${ }^{\mathrm{TM}}$ ): Little infrared markers were attached to index fingers and thumb and allowed via special video cameras 
to determine the trajectories of the grasping movements.

When grasping, index finger and thumb open to a maximum grip aperture (MGA) before touching the grasp object (Figure 1d). The MGA is linearly related to the size of the target object (Jeannerod, 1981, 1984) and is therefore interpreted as an index of the size information in the action system. The MGA was the dependent variable in all example studies discussed in this article. The advantage of using the MGA is that it is formed before the hand has any contact with the target object. Therefore, it is not contaminated by mechanical interactions of the fingers with the object. The MGA probably has the function of providing a "safety margin" when moving the fingers into an optimal position to close down around the target object, and it therefore is always larger than the actual size of the target object (this is relevant if we wanted to calculate percentages, see: "Appendix A: Why do researchers report illusion effects in millimetres instead of percentages?"). Effects of the Ebbinghaus illusion on grasping, as shown in Figure 1e, were calculated as the difference: MGA in small-near condition minus MGA in large-far condition.

In most example studies, grasping was performed open loop. That is, as soon as the fingers started the reach-to-grasp movements, goggles prevented vision of hand and stimuli during the movement. This was done to prevent the participants from performing online corrections during the movements, which might be based on visual feedback and therefore might contaminate the data (Post \& Welch, 1996). It turned out, however, that the two studies that used closedloop grasping (Aglioti et al., 1995, and Pavani et al., 1999) came to very similar results as the studies that used open-loop grasping. Therefore, the issue of visual feedback does not seem very critical for the Ebbinghaus illusion and is not discussed in further detail here (for further discussions see Franz, Scharnowski, \& Gegenfurtner, 2005, and Haffenden \& Goodale, 1998). Note, however, that this is only true for the Ebbinghaus illusion. For example, when grasping the Müller-Lyer illusion (Figure 2d) there are
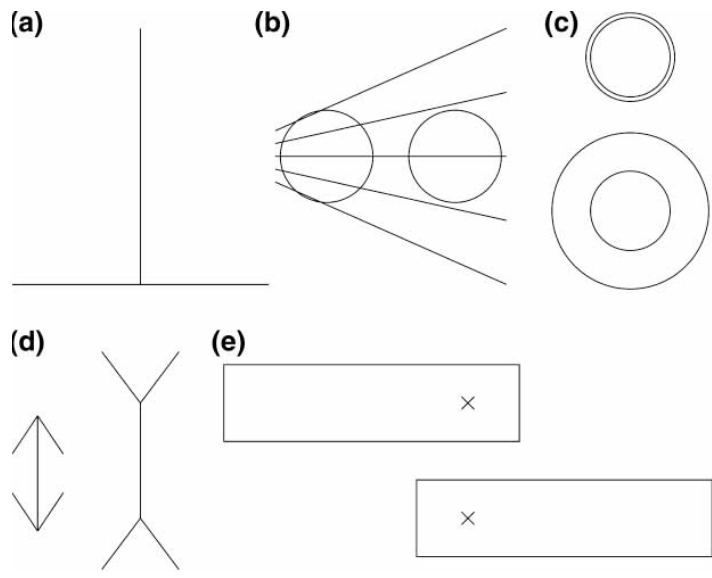

Figure 2. Some illusions used in perception-action studies. (a) Horizontal-vertical illusion (Fick, 1851). The horizontal line and the vertical line have the same length, but the vertical line appears longer. (b) Ponzo illusion (Titchener, 1901). The circles have the same diameter, but the circle that is closer to the (theoretical) apex appears larger. (c) Delboeuf illusion (Delboeuf, 1865; Nicolas, 1995). The upper central circle surrounded by a relatively small concentric circle appears larger than the lower central circle surrounded by a larger concentric circle. (d) MüllerLyer illusion (Müller-Lyer, 1889). The shafts have the same length, but the shaft with the outward pointing fins appears larger. (e) Induced Roelofs effect (Bridgeman et al., 1997; Roelofs, 1935). The crosses are vertically aligned, but the frame offset to the left makes the cross appear farther to the right.

big differences between open- and closed-loop grasping (e.g., Franz, Hesse, \& Kollath, 2007; Westwood, Heath, \& Roy, 2000c; Westwood, McEachern, \& Roy, 2001). In this case, the "better" condition for a comparison between perception and action is the open-loop condition (Post \& Welch, 1996).

To measure the effects of the Ebbinghaus illusion on perception, researchers used different types of measure. Most of the example studies used "classic" perceptual measures (Aglioti et al., 1995; Franz et al., 2003; Franz et al., 2000; Pavani et al., 1999): A comparison object is adjusted or selected to match the size of the target object. There are subtle differences between the classic perceptual measures: Aglioti et al. (1995) used what we call a "direct" comparison (and which is denoted as "Perc: direct" in the 
figures); participants directly matched two discs, one placed in the large-far configuration and the other in the small-near configuration. The difference between the matched discs was used as the measure of the illusion effect. Pavani et al. (1999), Franz et al., 2003, and Franz et al., 2000, used "separate" comparisons (denoted as "Perc: separate" in the figures): They placed a neutral comparison object outside either the large-far or the small-near configurations, and participants matched this comparison object to the size of the central disc. The illusion effect was determined (as in grasping) by calculating the difference: matched size in small-near condition minus matched size in large-far condition. The distinction between "direct" and "separate" comparisons has important consequences for the interpretation of the illusion effects. These are discussed below.

Two of the example studies (Haffenden \& Goodale, 1998; Haffenden et al., 2001) used another measure to assess perception: Participants used index finger and thumb to indicate the size of the central disc without seeing their fingers. The illusion effect was then determined (as in grasping) by calculating the difference: estimated size in small-near condition minus estimated size in large-far condition. This measure is often called "manual estimation" (denoted as "Man Est" in the figures) and assumed by Goodale, Haffenden, and colleagues to be a kind of manual "read-out" of what the participants perceive (Haffenden \& Goodale, 1998, p. 125). Manual estimation is therefore assumed to tap the vision-for-perception system but not the vision-for-action system. Here, we do not need to discuss whether this is a plausible assumption (we tend to be sceptical), because we argue that all measures, manual estimation, classic perceptual measures, and grasping are affected in a similar way by the Ebbinghaus illusion, such that this question is not very relevant. Note, that manual estimation has been used by a large number of studies that found a dissociation between perception and action also in other visual illusions and is therefore an important measure to be discussed here (e.g., Bartelt \& Darling, 2002; Daprati \& Gentilucci, 1997; Otto-de Haart, Carey, \&
Milne, 1999, Westwood, Chapman, \& Roy, 2000a; Westwood, Dubrowski, Carnahan, \& Roy, 2000b; Westwood et al., 2001).

Having described how illusion effects were measured, we can now proceed to answer our two questions: (a) If we assume that the perception-versus-action hypothesis is correct, then why is there an illusion effect on grasping at all? (b) Which measure of the perceptual illusion should be compared to grasping? We discuss these questions successively.

\section{Why is there an effect of the illusion on grasping at all?}

To reconcile the finding of illusion effects in grasping (Figure 1e) with the perception-versusaction hypothesis, Haffenden, Goodale, and colleagues suggested that the illusion effects on grasping are generated in the vision-for-action system by different mechanisms from those for the perceptual effects (Haffenden \& Goodale, 2000; Haffenden et al., 2001; Plodowski \& Jackson, 2001). They argued that the context circles of the Ebbinghaus illusion are treated as obstacles for the fingers and therefore affect the trajectories of the grasp movements. Because the eccentricity of the context circles was not equated in the Ebbinghaus figures of the example studies (Figure 1a), the effects on grasping would be artefacts, and what might look like an illusion in the action system might be due to different mechanisms from those for the perceptual illusion. Therefore, this explanation could reconcile the effects of the Ebbinghaus illusion on grasping even with a strict perception-versus-action framework in which grasping is not deceived by the Ebbinghaus illusion at all. This notion bears, however, two problems:

First, Haffenden et al. proposed an implausible obstacle avoidance mechanism. To see this, consider the Figures $3 \mathrm{a}$ and $3 \mathrm{~d}$, which show the standard versions of the Ebbinghaus illusion as were used in the example studies. In the large-far condition the central disc is perceived as being smaller than that in the small-near condition. Haffenden et al. (Haffenden \& Goodale, 2000; Haffenden 


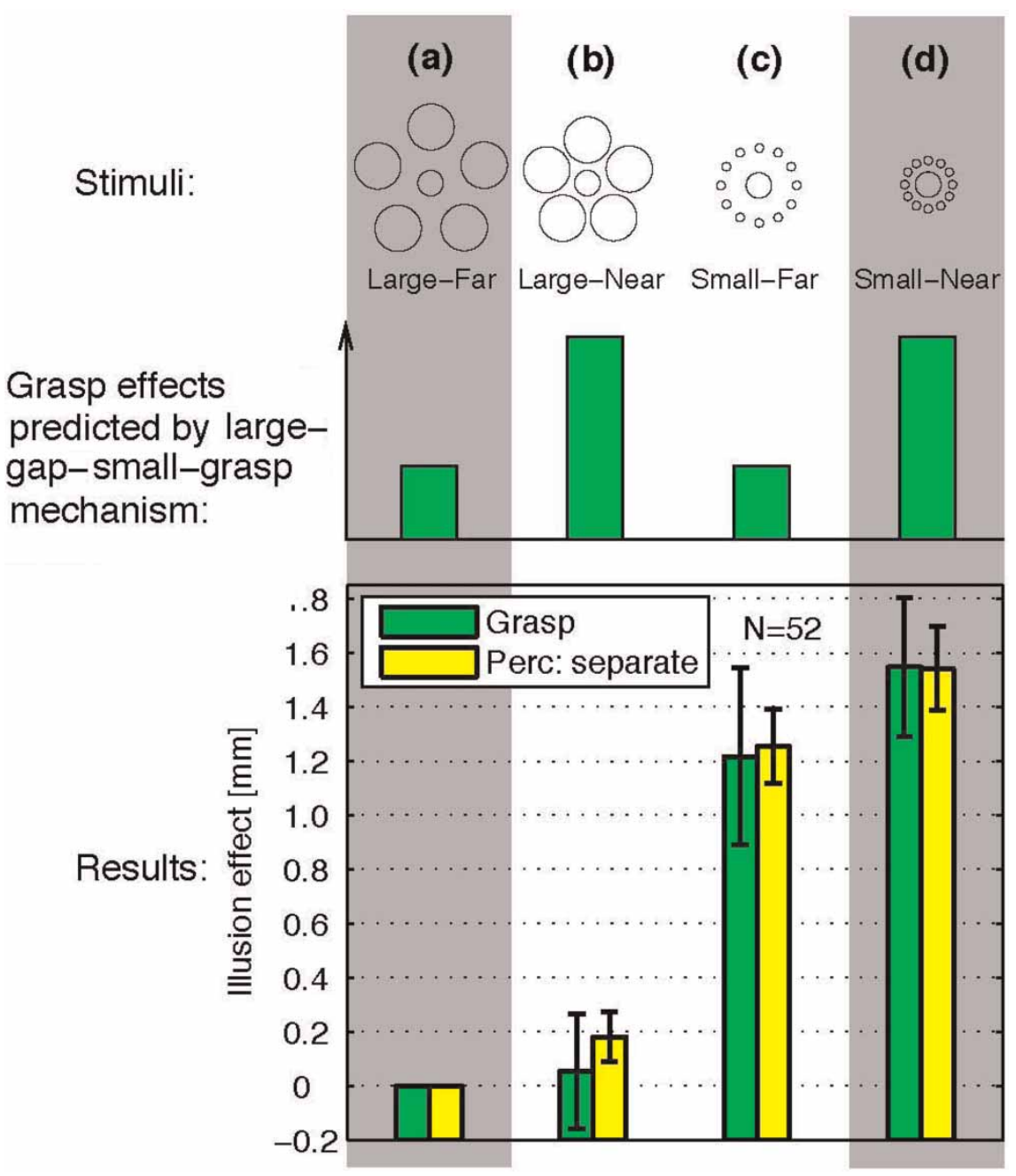

Figure 3. Testing for obstacle avoidance mechanisms. Franz et al. (2003) tested the obstacle avoidance mechanism proposed by Haffenden et al. (2001), which states that a large gap between central disc and context circles leads to smaller grip apertures than does a small gap (large-gapsmall-grasp mechanism). Post hoc, this mechanism can explain the results of the sample studies that we also replicated in this study, as shown in the grey shaded areas (a) and (d); these results are also shown in Figure 1e as: "Franz 03". However, if we vary the distances of the context circles, then the large-gap-small-grasp mechanism leads to predictions for grasping that are opposite to the measured grasp effects (b) and (c). Therefore, the large-gap-small-grasp mechanism fails to explain the grasp effects of the Ebbinghaus illusion. Instead, grasping follows nicely the illusion found in perception. As in Figure 1, the illusion effects are calculated as the mean differences relative to the large-far condition (e.g., in the small-far condition, the illusion effect is the measured value in the small-far condition minus the measured value in the large-far condition). All error bars depict \pm 1 SEM. From "Grasp Effects of the Ebbinghaus Illusion: Obstacle-Avoidance is Not the Explanation", by V. H. Franz, H. H. Bülthoff, and M. Fahle, 2003, Experimental Brain Research, 149, pp. 470-477, Figure 5. Copyright 2003 by Springer Science and Business Media. Adapted with permission. (Figure can be seen in colour online.)

et al., 2001) proposed that this effect is solely due to obstacle avoidance relative to the context circles. How could such a mechanism work? (Note, that the context circles were always only two dimensional, such that there was no physical need to perform obstacle avoidance; for simplicity, let us assume that the action system nevertheless performs obstacle avoidance relative to the 
context circles.) Simple, straightforward obstacle avoidance mechanisms would predict that either participants grasp larger in the overall larger configuration or in the configuration with a greater gap between central disc and context circles. In both cases this would be the large-far condition. However, we already know from the example studies that participants do not do this, but grasp smaller in this configuration. To explain this, Haffenden et al. needed to assume an inverse relationship for obstacle avoidance: In the largefar condition participants are assumed to fit their fingers in the greater gap between central disc and context circles (like in a hole) and consequently are assumed to grasp smaller, while in the small-near condition, this is assumed to be not possible because the gap is too slim, and therefore participants grasp larger (around the whole small-near figure). Only this inverse relationship (large gap leads to smaller grasp) could explain the data of the example studies. Therefore, this "large-gap-small-grasp mechanism" relies on quite specific assumptions, which are not supported by the visuomotor literature on obstacle avoidance (e.g., Mon-Williams, Tresilian, Coppard, \& Carson, 2001; Schindler et al., 2004; Tresilian, 1998).

Second, we tested the large-gap-small-grasp mechanism and found clear evidence against it (Franz et al., 2003). For this, we created two new conditions by varying the gap sizes such that the predictions of the large-gap-small-grasp mechanism were opposite to the perceptual effects of the illusion (Figures $3 b$ and $3 c$ ): We created the large-near condition by placing the large context circles nearer to the central disc and the small-far condition by placing the small context circles further away. According to the large-gap-small-grasp mechanism this should lead to an inversion of the obstacle avoidance effects on grasping: Now participants should grasp larger in the large-near condition than in the small-far condition (see the predictions for grasping in the second row of Figure 3). For the perceptual illusion, however, we expect no such inversion, but only a slight attenuation of the illusion effect (Girgus et al., 1972). Our results clearly show that grasping closely follows perception and not the prediction of the large-gap-small-grasp mechanism (see the third row of Figure 3). These results provide strong evidence against the large-gap-small-grasp mechanism.

The earlier study of Pavani et al. (1999) can also be seen as an incidental test of the large-gap-smallgrasp mechanism: They compared a condition with large context elements to a neutral condition with context elements of equal size as the central element. The gaps were relatively large in both conditions $(28 \ldots 32 \mathrm{~mm})$, larger than the critical gap size $(3 \ldots 11 \mathrm{~mm}$, see below) proposed by Haffenden et al. (Haffenden \& Goodale, 2000; Haffenden et al., 2001), such that the large-gapsmall-grasp mechanism should not produce any effect. But even if we assume that the large-gapsmall-grasp mechanism is activated by these stimuli, then it should have produced smaller grasping in the neutral condition, because here the gaps were by $2 \ldots 5 \mathrm{~mm}$ larger than those in the condition with large context elements. But this was not the case: Participants grasped larger in the neutral condition, and this effect was very similar to the perceptual effect produced by these stimuli. This also constitutes evidence against the largegap-small-grasp mechanism.

Haffenden et al. also tested the large-gapsmall-grasp mechanism in two studies. We think, however, that these studies do not provide strong evidence for the large-gap-small-grasp mechanism: In the first study Haffenden and Goodale (2000) reanalysed their data of Haffenden and Goodale (1998) and derived from this the large-gap-small-grasp hypothesis. They suggested that with a relatively large gap of $11 \mathrm{~mm}$ between central disc and context circles, participants should grasp small, while with a small gap of $3 \mathrm{~mm}$ they should grasp large. Based on this idea, they flanked a disc with rectangles at different distances in order to "directly test whether the flanking objects alone could produce an effect on grasp analogous to that seen with the Ebbinghaus display" (p. 1601). In a "horizontal" condition, the flanking rectangles were adjacent to the contact points for the grasp and therefore were assumed to activate the 
large-gap-small-grasp mechanism. In a "vertical" condition, the flanking rectangles were rotated by 90 degrees, such that they should not interfere with the grasp. Haffenden and Goodale (2000) interpreted their results as supporting the largegap-small-grasp hypothesis: "As we had predicted, there was a small reduction in grip aperture when flankers were placed adjacent to contact points but with a finger-sized gap between the target and the flanking elements. Thus for the $11 \mathrm{~mm}$ flanker distance, grip scaling was reduced for the horizontally oriented flankers relative to the vertically oriented flankers" (p. 1604).

However, none of the critical effects on grasping were statistically significant: The predicted interaction between distance of the flankers and the vertical/horizontal flanker orientation was not significant, $F(3,33)=1.39, p=.26$ (p. 1603). Next, Haffenden and Goodale (2000) performed more specific analyses and concentrated only on the flanker distances they had identified as being critical ( $3 \mathrm{~mm}$ and $11 \mathrm{~mm}$ ) and excluded the other flanker distances they had used $(21 \mathrm{~mm}$ and $31 \mathrm{~mm}$ ). But again, the predicted interaction between distance of the flankers and the vertical/ horizontal flanker orientation was not significant, $F(1,11)=3.71, p=.08$ (p. 1604). Finally, they focused only on the 11-mm flanker distance. But again, they only found a trend in the predicted direction, which was clearly not significant, $t(11)$ $=1.34, p=.21$ (Fig. 6, p. 1603). To support their conclusions, Haffenden and Goodale (2000) also referred to a replication of this experiment, which was presented at a conference (Haffenden, Zavitz, \& Goodale, 2000), but admit that here the critical effect was also not significant: "And again, at the critical $11 \mathrm{~mm}$ flanker distance, a small, non-significant, effect was seen on grasp-consistent with the idea that subjects were paring down their fingers to avoid the flankers placed adjacent to contact points" (p. 1605).

Now, one might argue that Haffenden and Goodale (2000) also measured perception using manual estimation. The main difference they found between grasping and manual estimation was that the flanker distance had a significant effect on manual estimation, while the effect on grasping was not significant (but they did not compare these effects, see "Appendix B: The significant/not-significant-as-difference fallacy" for why we should not infer a difference from a nonsignificant effect on grasping and a significant effect on manual estimation). However, for a demonstration of the large-gap-small-grasp mechanism, perceptual measures are of minor importance because by definition the large-gapsmall-grasp mechanism should be independent of perception. Given that all the effects predicted by the large-gap-small-grasp mechanism for grasping were not significant, we think that this study does not provide evidence for the proposed large-gap-small-grasp mechanism.

In the second study, Haffenden et al. (2001) performed a similar test to that in Franz et al. (2003). However, they included only the small-far condition and not the large-near condition. According to the large-gap-small-grasp mechanism participants should grasp similarly in the small-far and the large-far conditions-and this is what Haffenden et al. (2001) found. However, this experimental design is based on a null effect and is therefore not as strong as our design, which predicts opposite effects for the competing hypotheses (for a power analysis showing that Haffenden et al., 2001, might have missed the effect due to insufficient power see Franz et al., 2003). Our interpretation is supported by the fact that Franz et al. (2003) found in grasping a highly significant illusion effect between the small-far and the large-far conditions, which we also replicated in Franz et al. (2005). Similarly, we also found a highly significant illusion effect between the large-near and small-near conditions, which we also replicated in Franz (2003a; these results are also shown in Figure 5). These results cannot be explain with the large-gapsmall-grasp mechanism.

In summary, it is unlikely that the large-gapsmall-grasp mechanism proposed by Haffenden et al. (Haffenden \& Goodale, 2000; Haffenden et al., 2001) does account for the effects of the Ebbinghaus illusion on grasping. Instead, grasping seems to respond in a similar way to small variations of the illusion as perception. 


\section{Which perceptual measure should be compared to grasping?}

In the last section we have argued that effects on grasping are probably "true" illusion effects; at least we did not find evidence that other mechanisms have created these effects in the vision-foraction system independent of perception. This rules out the strong version of the perceptionversus-action hypothesis, which assumes that there is no effect of the Ebbinghaus illusion on grasping at all. But, nevertheless, we might opt for a weak version of the hypothesis, claiming that grasping is less affected by the illusion than is perception. To test such a hypothesis, we have to compare the sizes of the illusion in perception and in grasping. This leads to the problem that three different measures were used for perception, each generating a different estimate for the perceptual illusion. Which of these perceptual measures should be compared to grasping? We first discuss the two classic perceptual measures (which employed either direct or separate comparisons) and argue that the separate comparisons are better suited to be compared to grasping. Then, we discuss manual estimation and argue that manual estimation, the classic perceptual measures with separate comparisons, and grasping are all similarly affected by the Ebbinghaus illusion, such that there is indeed no dissociation between perception and grasping in this illusion.

\section{Classic perception: Direct comparison or separate comparisons?}

Pavani et al. (1999) and we (Franz et al., 2003; Franz et al., 2000) used separate comparisons to asses the perceptual effects of the illusion, while Aglioti et al. (1995) used a direct comparison. For the separate comparisons, participants always operated on only one Ebbinghaus figure at a time and either grasped the central disc or adjusted a neutral comparison circle to match the size of the central disc (Figure 4b). In contrast, Aglioti et al. (1995) presented a composite version of the illusion, consisting of two Ebbinghaus figures (Figure 4a). In grasping, participants successively operated on only one of the Ebbinghaus figures and grasped one of the central discs. In perception, however, they performed a direct comparison between the two central discs, both being subjected to the illusion at the same time. Therefore, there is a mismatch in task demands between grasping and perceptual task in the Aglioti et al. study.

It turned out that this mismatch is critical (Franz et al., 2000). In a first step, we showed that we could replicate the difference in the perceptual measures between our studies and the Aglioti study (Figure 4c): The direct comparison leads to an approximate $50 \%$ increase of the illusion compared to the sum of the separate comparisons. That is, the Ebbinghaus illusion is overadditive: The measured illusion effect in the direct comparison is more than the sum of the illusion effects in the two separate comparisons. This is not surprising given that the attentional demands are quite different in these tasks. It seems likely that the increased illusion effect is caused by an interaction of the context elements of the illusion in the direct comparison (i.e., the large context elements might appear as even larger if they are contrasted directly with the small context elements; see also Pavani et al., 1999, for a discussion of this issue).

In a second step, we mimicked the procedure of Aglioti et al. (1995) in which participants always first compared the two central discs and then grasped one of the discs. But, instead of grasping, we had participants perform a perceptual task with similar task demands to those in grasping: In a constant stimuli procedure participants viewed two Ebbinghaus figures and first compared the two central circles (as they did in Aglioti's perceptual task) and then compared one of the central circles to a neutral circle, positioned outside the two Ebbinghaus figures (this mimicked Aglioti's grasping task, because participants operated on only one of the central circles at a time). Both tasks were performed in rapid succession within 1 s. As can be seen in Figure 4d, even this rapid succession of the tasks leads to the $50 \%$ increase of the illusion in the direct comparison. This shows that the difference that Aglioti et al. found between perception and grasping can be replicated in purely 
(a)

Aglioti 95 (b)

Pavani 99

Franz (c)

Franz 00 (d)

Franz 00 (mimic Aglioti 95 in perception)

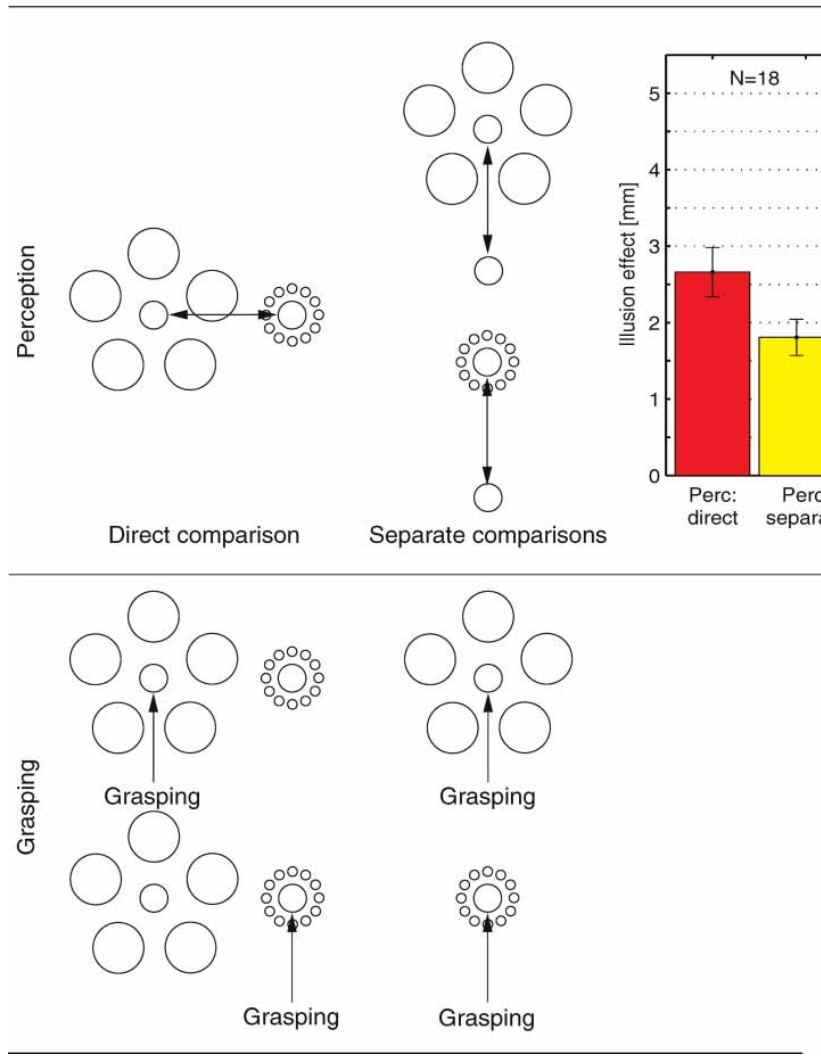

First: Direct comparison...

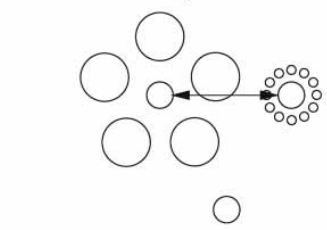

... then: Separate comparison (mimics grasping)
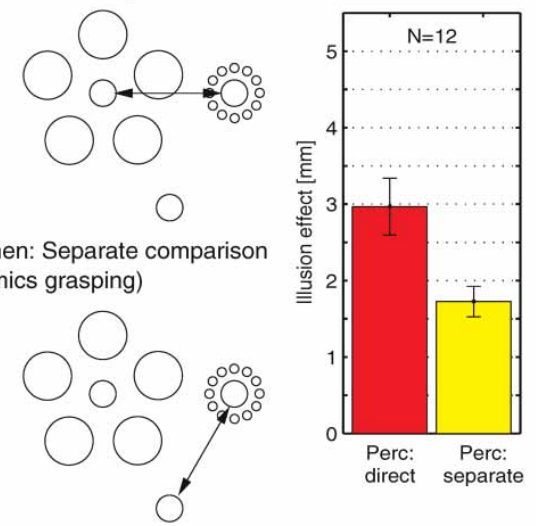

Figure 4. Task demands in perceptual and grasping tasks. Comparison of the task demands in Aglioti et al. (1995), Pavani et al. (1999), Franz et al. (2003), and Franz et al. (2000). (a) In the Aglioti study, two Ebbinghaus figures were shown. In the perceptual task participants compared the two central discs directly, both being subjected to the illusion at the same time. In the grasping task, however, they grasped one or the other of the discs, thereby needing to calculate only the size of one of the central discs. Note the asymmetry in this procedure. (b) In Pavani's and our studies, participants always operated on only one Ebbinghaus figure at a time in grasping as well as in perception (separate comparisons). Therefore, the tasks were better matched than in the Aglioti et al. study. (c) Franz et al. (2000) showed that the different task demands in the Aglioti study led to larger illusion effects even in perceptual tasks: The direct comparison increases the measured illusion effect by approximately 50\% as compared to the sum of the illusion effects in the separate comparisons. This is the difference that Aglioti et al. found between perception and grasping. (d) The difference between direct and separate comparisons persists even if participants always see both illusion figures and perform direct and separate comparison in rapid succession (Franz et al., 2000): In a constant stimuli procedure, participants first compared the two central circles and then compared one or the other of the central circles to a neutral circle outside the display (both comparisons were performed together within $1 \mathrm{~s}$ ). The direct comparison closely mimics the perceptual task in the Aglioti et al. study and the separate comparisons the grasping task. Again, we find the approximately 50\% increase of the illusion effect in the direct comparison relative to the sum of the illusion effects in the separate comparisons. This suggests that the differences that Aglioti et al. found between perception and grasping (Figure 1e) are due to the different task demands and not to a dissociation between perception and action. "Perc: direct" and "Perc: separate": Classic perceptual measures for direct and separate comparisons, respectively. Data in (c) are from Franz et al. (2000, Exp. 2; conditions "sum of single" and "direct"), and data in (d) are from Franz et al. (2000, Exp. 3). All error bars depict \pm 1 SEM. From "Grasping Visual Illusions: No Evidence for a Dissociation Between Perception and Action”, by V. H. Franz, K. R. Gegenfurtner, H. H. Bülthoff, and M. Fable, 2000, Psychological Science, 11, pp. 20-25, Figure 5. Copyright 2000 by Blackwell Publishing. Adapted with permission. (Figure can be seen in colour online.) 
perceptual tasks and is therefore probably due to the different task demands and not to a difference between perception and action.

These experiments also show that requiring a perceptual judgement first and then the grasp immediately after (as suggested by Carey, 2001) cannot be expected to increase the illusion effect in grasping to the level of the direct comparison in perception. This is so, because even in purely perceptual tasks a rapid succession of direct comparison and separate comparison does not increase the illusion effect in the separate comparison to the level of the direct comparison. Therefore, there is no reason to expect this to happen in grasping.

The distinction between direct and separate comparisons is also relevant for studies on other visual illusions. For example, Vishton, Pea, Cutting, and Nunez (1999) investigated the horizontal-vertical illusion (Figure 2a). They demonstrated a much larger illusion effect if the horizontal-vertical illusion was measured with a direct comparison than if it was measured with separate comparisons (which they called relative and absolute measures, respectively). This was true, independent of whether the task was perceptual or an action. Vishton et al. (1999) therefore came to the same conclusion for the horizontal-vertical illusion as we did for the Ebbinghaus illusion: It is the task demands and not the response mode that leads to a larger illusion effect in the direct comparison. Further studies for which this distinction is important are the studies by Brenner and Smeets (1996) and Jackson and Shaw (2000) on the Ponzo illusion (Figure 2b). These are discussed below.

In summary, classic perceptual measures that are based on a direct comparison between two stimuli that are both subjected to the illusion are not well matched to grasping because grasping typically operates successively on only one of the two stimuli. In the Ebbinghaus illusion, the direct comparison leads to an approximately 50\% increase of the illusion, which corresponds well to the difference that Aglioti et al. (1995) found between perception and grasping (Figure 1e). Therefore, this difference is better explained by a mismatch in tasks demands than by a dissociation between perception and action.

\section{Classic perception or manual estimation?}

Haffenden and Goodale (1998) suggested manual estimation as another measure to determine the perceptual illusion: Participants indicated the size of the central disc of the Ebbinghaus figure by using index finger and thumb (without seeing the hand). This measure is based on neuropsychological considerations and has been used very often in studies on perception-action dissociations. As mentioned above, Haffenden and Goodale (1998) interpret manual estimation as a purely perceptual measure, which provides a manual readout of the vision-for-perception system. Given its wide use it seems quite surprising that the measure had not been tested against classic perceptual measures as, for example, adjustment procedures in which participants adjust the size of a neutral comparison to match the size of the target object. Therefore, it was not clear whether manual estimation would yield similar results to those for classic perceptual measures. Franz (2003a) performed such a validation study measuring the illusion effects for manual estimation, classic perceptual measures, and grasping. The study roughly replicated the differences found between the example studies (compare Figure $5 \mathrm{a}$ and Figure 1e). Most importantly, manual estimation showed again the largest illusion effects.

Interestingly, an analysis of the response functions revealed that manual estimation reacted to any change of object size stronger than the other measures. We found that if the physical size of the target was increased by $1 \mathrm{~mm}$, then manual estimation reacted by an increase of $1.6 \mathrm{~mm}$. That is, the slope of the linear function relating physical size to manual estimation was 1.6. This is consistent with other studies. For example, Haffenden et al. (2001) reported an even larger slope of 1.85 for manual estimation. Classic perceptual measures, however, typically have a slope of approximately 1 , while the MGA in grasping has a slope of approximately 0.82 (Smeets \& Brenner, 1999).

Accordingly, we expect that an illusory change of object size will also affect manual estimation more than the other measures. To see this, 


\section{Franz 03a}

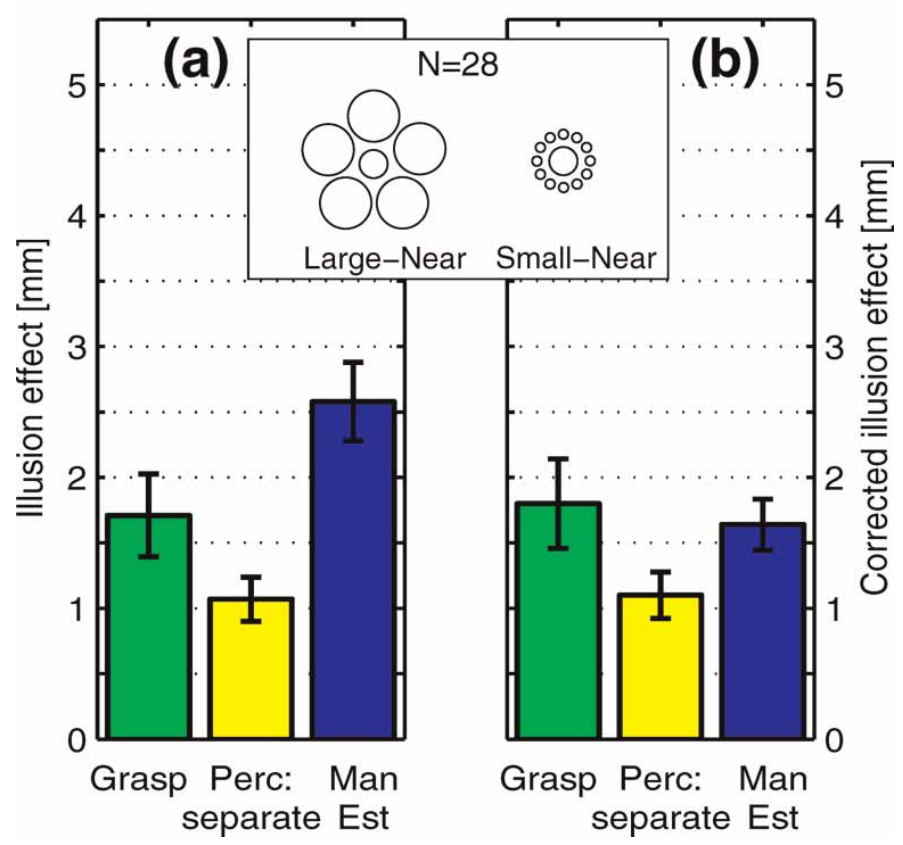

Figure 5. Manual estimation and response functions. (a) Franz (2003a) replicated the differences found in the example studies between grasping, manual estimation, and classic perceptual measures. (b) However, an investigation of the response functions, relating physical size to the dependent measures, shows that manual estimation depends on physical size with a higher slope. Therefore, we have to correct for these different slopes. This is done by dividing each illusion effect by the slope of the corresponding response function. After correction, the differences between the illusion effects get much smaller. "Grasp": MGA when grasping. "Perc: separate": classic perceptual measure for separate comparisons. "Man Est": manual estimation. All error bars depict \pm 1 SEM. From "Manual Size Estimation: A Neuropsychological Measure of Perception?", by V. H. Franz, 2003, Experimental Brain Research, 151, pp. 471-477, Figure 3. Copyright 2003 by Springer Science and Business Media. Adapted with permission. (Figure can be seen in colour online.)

consider the case that we introduced an illusion of, say, $2 \mathrm{~mm}$. This should lead in manual estimation to a change of $2 \times 1.6 \mathrm{~mm}=3.2 \mathrm{~mm}$, but in classic perception only to a change of $2 \times 1 \mathrm{~mm}=2 \mathrm{~mm}$ and in grasping to a change of $2 \times 0.82 \mathrm{~mm}=$ $1.64 \mathrm{~mm}$. Consequently, we have to correct for the different slopes of the response functions. The correction is done by dividing the measured illusion effect by the slope (cf., Franz, 2003a; Franz, 2007; Franz, Fahle, Bülthoff, \& Gegenfurtner, 2001; Franz et al., 2005; Glover \& Dixon, 2002). If we do this, the differences between manual estimation, classic perceptual measures, and grasping disappear (Figure 5b).

We can also perform the correction for the data of Haffenden et al. (2001) as shown in Figure 1e. If we perform the correction, this again leads to similar illusion effects for manual estimation and grasping (Figure 1f).

It is interesting to speculate why manual estimation is so responsive to a (physical or illusionary) variation of size. Because no visual feedback of the hand is allowed during performance of the task, participants have to rely exclusively on proprioceptive feedback. It is likely that proprioceptive information is not as accurate as visual information, and therefore participants might exaggerate their response (Franz, 2003a). This reasoning also shows that the interpretation of manual estimation as a purely perceptual measure is not as obvious as has often been assumed: While indicating the size of an object with index finger and thumb (without seeing the hand), participants must use proprioceptive cues and employ 
a number of motor processes. It is not clear why these motor processes should not affect the outcome of the measure (cf. Pavani et al., 1999, for a similar argumentation). In fact, some authors used responses similar to manual estimation to assess motor effects of visual illusions (e.g., Vishton et al., 1999), or to investigate more general aspects of the visuomotor transformation (Jeannerod \& Decety, 1990). This interpretation is consistent with the finding that the slope of manual estimation seems to be closer to 1 if full vision of the hand is allowed during manual estimation (Dewar \& Carey, 2006; Otto-de Haart et al., 1999; Radoeva, Cohen, Corballis, Lukovits, \& Koleva, 2005).

In summary, manual estimation turned out to be roughly twice as responsive to a change of physical object size as was grasping (slopes of 1.6 or 1.85 vs. slopes of 0.82 ). Therefore it is no surprise that manual estimation also responded to an illusionary change of object size much more than did grasping. If we correct for the different slopes, we obtain similar illusion effects in manual estimation, grasping, and classic perceptual measures.

\section{Preliminary summary}

We argued that the grasp data on the Ebbinghaus illusion are surprisingly consistent. The perceptual data are not as consistent. However, if we use such methodological precautions as taking into account the slope of the measures' response functions and making sure that all tasks are appropriately matched, then we can explain the differences between classic perceptual measures, manual estimation, and grasping. In consequence, we find that all these measures are similarly affected by the Ebbinghaus illusion.

But, how general and valid is this result? In the following sections, we discuss issues raised in the literature and by reviewers. First we discuss some direct critique that has been raised against our arguments ("More detail 1: Critique of our arguments"), then the question of which mechanisms lead to the Ebbinghaus illusion ("More detail 2: How is the Ebbinghaus illusion generated?"), then alternative experimental paradigms and illusions ("More detail 3: Other paradigms and illusions"), and finally other theoretical accounts ("More detail 4: Alternative theoretical accounts"). All these issues require that we go into more detail, such that a reader not interested in these more indepth discussions might want to jump to the "General Discussion" to get an impression of the main thrust of our argument. We also added an Appendix in which we discuss more technical issues that might help a researcher to conduct studies in this field but that might distract the reader if included in the main text.

\section{More detail: 1. Critique of our arguments}

In this section we discuss specific critique that has been raised against the validity of our conclusions.

\section{You failed to replicate the Aglioti study}

Goodale, Kroliczak, and Westwood (2005; p. 278) and Goodale and Westwood (2004; p. 206) argued that we failed to replicate the original study of Aglioti et al. (1995), thereby implying that we might have missed an existing effect. But, this is not the case: In Franz et al. (2000) we replicated Aglioti et al.'s effects of the Ebbinghaus illusion on grasping (cf. Figure 1), as well as their effects on perception (cf. Figure 4). However, we showed that the differences they found between grasping and perception are probably due to different task demands and not to a dissociation between action and perception.

\section{You concentrate on only half of the trials in the Aglioti study and ignore the other half}

Goodale (2006) argued that we use only half of the trials of the Aglioti et al. (1995) study for our comparison. This is indeed the case: The illusion effects shown for perception and grasping in Figure 1e for Aglioti et al. (1995) are based on only half of the trials. They correspond to the values of the Figure 5 of Aglioti et al. (1995), for which the figure legend says that it shows "the effect of the illusion on grip size". Due to the experimental design of Aglioti et al. (1995), it is not possible to quantitatively estimate the illusion 
effects from the other half of the trials without additional assumptions (because in these trials the sizes of context elements and of central disc were confounded). Therefore, Aglioti et al. (1995) used only half of the trials to calculate a quantitative measure of the illusion effect and did not give any numbers for the illusion effect in the other half of the trials that we could use. Note, however, that this is not very critical, because (a) there is no reason why the illusion effects should be different in the other half of the trials (the only difference was that the central discs had slightly different sizes), and (b) the later studies found similar effects of the illusion on grasping without having these problems (cf. Figure 1e).

\section{Separate comparisons reduce the illusion too much}

Some researchers expressed concern that using separate comparisons (i.e., only one Ebbinghaus figure at a time) reduces the illusion too much. Jacob and Jeannerod (1999) argued that we "used a display in which the perceptual illusion does not properly arise .... So when ... a stimulus is not a suitable basis for producing the perceptual illusion, it should not be surprising to find that the motor response is not influenced by the illusion either. Nor should it be surprising to find that the perceptual response and the motor response to the non-illusory stimulus do not really differ. In fact, if a stimulus does not give rise to a perceptual illusion, it does not provide an adequate basis for drawing any conclusion upon Goodale and Milner's hypothesis" (p. 9; see also Carey, 2001, p. 109). We think, however, that this argument is not valid because we and other researchers found perceptual and motor illusions of approximately 4-5\% (cf. Figure 1e and "Appendix A: Why do researchers report illusion effects in millimetres instead of percentages?"). This is in agreement with the effects found in the perceptual literature (although, of course, a composite version of two Ebbinghaus figures and a direct comparison between these figures would lead to a larger illusion, as we have shown in Franz et al., 2000). We even can discriminate very small variations of the illusion in perception and grasping by varying the distance of the context elements slightly (cf. Figure 3 and Franz et al., 2003). Also note that it is standard procedure in perceptual research to use only one Ebbinghaus figure at a time- as we did in our separate comparisons (e.g., Choplin \& Medin, 1999; Coren \& Enns, 1993; Coren \& Girgus, 1972; Coren \& Miller, 1974; Girgus et al., 1972; Jaeger, 1978; Jaeger \& Pollack, 1977; Massaro \& Anderson, 1971; Pavlova \& Sokolov, 2000; Pressey, 1977; Roberts, Harris, \& Yates, 2005; Weintraub, 1979; Weintraub \& Schneck, 1986). This standard procedure leads to a better control of the stimulian advantage we certainly also want to exploit in studies on perception and action.

\section{You measured vision-for-perception but not vision-} for-action in grasping

Goodale (2006) suggested that-due to some specifics of our grasping task-we erroneously measured vision-for-perception with our grasping task but not vision-for-action. Consequently, it would be no surprise that we found similar effects of visual illusions on grasping and on perception.

This argument is based on the fact that we used a different method to attach the infrared markers to the fingers from that used in the studies of Aglioti and Haffenden (Aglioti et al., 1995; Haffenden \& Goodale, 1998; Haffenden et al., 2001). The idea is that our method let to awkward grasping, which might make the right hand behave like an unskilled left hand. Because Gonzalez, Ganel, and Goodale (2006a) argued that the left hand is controlled by the vision-for-perception system (see the discussion below), this would mean that we never measured vision-for-action with our grasping task, but always vision-for-perception. In consequence, it would be no surprise that we found illusion effects in our grasping task.

An example of our method can be seen in Figure 1b: We attached to the finger-nails of index finger and thumb small, lightweight aluminium flags, each with three infrared markers. Aglioti and Haffenden on the other side, attached only one marker to each finger (Figure 1c). Our method has two advantages: (a) Employing mathematical rigid-body transformations on the three 
markers, we determined the trajectories of the grasp points for each finger. This is not possible with the methods of Aglioti and Haffenden. Therefore they always had an additional measurement error, depending on the thickness and orientation of the fingers. (b) With our method the finger tip is completely free, allowing for full tactile feedback. This is not guaranteed with the method of Aglioti and Haffenden because the tape they used to attach the single marker could cover parts of the finger tip (as can be seen in Figure 1c).

But, what about the awkwardness? Of course, when running the experiment we tried to make sure that the markers (and cables) did not interfere with the natural grasping movement. But, maybe this was not sufficient to ensure natural grasping? There are two reasons why we think the conjecture of Goodale (2006) is not valid: (a) If it were correct, then our results should be "atypical". That is, we should obtain larger illusion effects for grasping than those in other studies. But this is not the case (Figure 1e). In fact, the grasping data are surprisingly consistent across all laboratories (this is one of the main messages of this review). (b) In a recent study, we tested the conjecture directly. We split our 40 participants in two groups using the two different methods to attach the markers and had them perform an identical experiment on the Müller-Lyer illusion. We did not find any difference of the illusion effects (Franz et al., 2007). This was the first direct empirical test of the conjecture-and the results refute it.

\section{More detail: 2. How is the Ebbinghaus illusion generated?}

Given the controversy on the dissociation between perception and action in the Ebbinghaus illusion it would be beneficial to know where exactly the illusion is generated. For example, we could ask whether the Ebbinghaus illusion is indeed generated in the ventral stream, as assumed by Aglioti et al. (1995) and Milner and Goodale (1995). Unfortunately, not much is known on the exact neuronal sources of the illusion. Roberts et al. (2005) conclude that since the late 1970s not much progress has been made on explaining the
Ebbinghaus illusion and that since then it has been mainly used as a research tool. In fact, researchers often took the Aglioti et al. (1995) result as an indication that the illusion might be created in ventral areas (Eagleman, 2001; Wade, 1998). Nevertheless, we give a short overview of the research on the sources of the Ebbinghaus illusion and show that some (but not all) of the research might be consistent with an early source of the illusion.

To explain the Ebbinghaus illusion, there are three main lines of argument in the literature: size contrast, other figural factors, and sizedistance arguments. We discuss these (not mutually exclusive) arguments successively.

\section{Size contrast}

Most theories describe the Ebbinghaus illusion as a size contrast effect: The size of an object that is surrounded by similar objects will be judged relative to the size of these objects. If the context elements are smaller, the central object will appear relatively large-and vice versa (Massaro \& Anderson, 1971; Restle \& Merryman, 1968). Critical for this effect is that the context elements are perceived as being similar to the central element (Coren \& Miller, 1974). While this mechanisms could in principle happen relatively early in the visual system, there was one study that suggested high-level contributions: Coren and Enns (1993) argued that a semantic shift between central element and context elements reduces the illusion-even if the low-level features of all objects are identical. Choplin and Medin (1999) however, criticized this finding and argued that Coren and Enns (1993) had confounded low-level features, as the similarity of the perimeters of the elements, with the semantic category. They argued, therefore, for an earlier source of the illusion.

\section{Other figural factors}

Other researchers stressed the fact that size contrast alone cannot explain all effects of the Ebbinghaus illusion - and might not even be the most important factor. One striking example is the finding that small context elements quite

\section{COGNITIVE NEUROPSYCHOLOGY, 2008, 25 (7-8)}


often reduce the size of the central element in comparison to a central element without any context elements (Girgus et al., 1972; Roberts et al., 2005). Therefore, other factors had to be taken into account. For example, Weintraub and Schneck (1986) suggested four further factors in addition to size contrast: (a) contour attraction: If the context elements are close to the central element this will lead to an attraction effect, thereby leading to an increase of the perceived size of the central element; with increasing distance, this will revert to a decrease of perceived size (even for smaller context elements); this mechanism is typically assumed to relate the Delboeuf (Figure 2c) and the Ebbinghaus illusions (Girgus et al., 1972; Roberts et al., 2005; Weintraub \& Schneck, 1986); (b) the orientation effect: Rotating the Ebbinghaus figure by $45^{\circ}$ can change the perceived size of the central element; (c) cardinal-axes framing effect: The larger the overall extent of the Ebbinghaus figure in horizontal or vertical direction the smaller the judged size of the central element; (d) figural aftereffect: Aftereffects of the central elements can change the perceived size of the central element if eye movements are allowed. We do not want to discuss these factors in more detail here. For our purpose it is only important that all these factors are based on relatively basic object features and therefore could operate on a relatively early level of the visual system.

\section{Size-distance arguments}

The third approach to an explanation of the Ebbinghaus illusion is based on the idea of size constancy (Day, 1972; Gregory, 1963; Thiéry, 1896; but see Coren \& Girgus, 1973, and McCready, 1985, for a critiques of this view). In its simplest form, the idea is that the small context elements are perceived as being relatively far away (because objects further away look smaller). This induces the corresponding central element to be perceived as being further away than if surrounded by large context elements. Because the central elements have the same retinal size the central element surrounded by small context elements is perceived as being larger than if surrounded by large context elements (this is Emmert's law; Emmert, 1881; Holway \& Boring, 1941). A similar explanation has been proposed for the Ponzo illusion: Here linear perspective leads to a difference in perceived distance and to the size illusion. Interestingly, Murray, Boyaci, and Kersten (2006) have shown that the effects of the Ponzo illusion seem to operate at a very early level. Using functional magnetic resonance imaging (fMRI) they showed that activity in the primary visual cortex (V1) is already dependent on perceived size of the object (and not on physical size), thereby opting for a very early generation of the Ponzo illusion. Further research might clarify whether this is also true for the Ebbinghaus illusion.

In summary, surprisingly little is known about the exact mechanisms and sources of the Ebbinghaus illusion. Some authors opt for a relatively early source of the illusion, while others suggest higher level contributions, partly based on the notion that only perception is affected by the illusion (which we are scrutinizing in this review). Note, also, that for our main argument (that grasping the Ebbinghaus illusion does not provide positive evidence for the perception-versus-action hypothesis) the exact source of the illusion is not as critical as for the argument of Milner and Goodale (1995). They have to assume that the illusion is generated in ventral areas, while all we are saying is that perception and grasping are similarly affected by the illusion-independent of where exactly the illusion is generated.

\section{More detail: 3. Other paradigms and illusions}

Up to now, we kept our discussion as close as possible to the original reasoning and paradigm used by Aglioti et al. (1995). In this section we try to gain a wider perspective by taking into account studies using alternative experimental paradigms or other visual illusions.

\section{One-handed versus two-handed grasping}

We have argued that for a "fair" comparison between perception and grasping we should use 
separate comparisons in the Ebbinghaus illusionbecause the perceptual illusion effect in the direct comparison is selectively enlarged (section "Classic perception: Direct comparison or separate comparisons?”). Two studies (Dewar \& Carey, 2006; Vishton \& Fabre, 2003) tried to solve this problem by using an alternative paradigm: They used bimanual grasping, in an attempt to match the task demands of grasping to the direct comparison in perception (and thereby employing the larger illusion also for the test of grasping). We think that this is an interesting approach. However, we see conceptual as well as empirical problems, such that we think that currently no strong conclusion can be based on these studies. Future research might help to come to a clearer picture here.

Let us first sketch our conceptual caveats: (a) It is not clear that bimanual grasping really introduces task demands similar to the direct comparison in perception. In bimanual grasping participants might very well be judging the absolute sizes of the two central discs independently (such that we would be at the level of two separate comparison again - with the corresponding smaller illusion effect). This could, for example, be the case if participants sequentially programme left and right hand independently (and thereby always attending to only one of the elements at a time, see also the discussion in Carey, 2001; Dewar \& Carey, 2006). (b) It should be ensured that the MGA of one hand responds to a variation of object size independently of the object size presented to the other hand. If, for example, due to computational limitations a "compromise" MGA were used for both hands, this might reduce an existing illusion effect. It might be possible to overcome this problem, though, because recent research on bimanual grasping has shown that it is possible to create conditions under which the MGAs of both hands respond quite independently to object size (Dohle, Ostermann, Hefter, \& Freund, 2000; Jackson, Jackson, \& Kritikos, 1999). This is, however, not always the case. If the two target object are perceptually unified to be part of a single object, then the MGAs become more similar across hands (Jackson,
German, \& Peacock, 2002), such that some caution is necessary here.

Now, let us describe our empirical caveats, which led us to argue that currently we cannot draw strong conclusions from these two studies: Vishton and Fabre (2003) used bimanual grasping in the Ebbinghaus illusion. However, they got quite mixed results, which are very hard to interpret. In Experiment 1 they found the largest illusion effects on one-handed and two-handed grasping (significantly larger than the effect on all their perceptual measures). They did not find significant illusion effect in one-handed and twohanded manual estimation and also not in one of their classic perceptual tasks (metric judgement: Participants verbally judged the size of the central discs in millimetres). Only in the comparative judgement task (participants verbally judged the size of one disc relative to the other) did they find a significant illusion effect. This result (largest illusion effect in grasping, hardly any illusion effect in perception) is highly unusual and runs counter to all the other results that were reported in the literature. In Experiment 2, they hypothesized that grasping of the discs was too difficult (because they might have been too thin) and placed the central discs on little posts $(4 \mathrm{~mm}$ high). Now, the illusion effect on grasping disappeared. In Experiment 3, they measured onehanded manual estimation with full vision of the hand during the task (while in Experiment 1 vision of the hand had been occluded during manual estimation). Now they obtained a similar illusion effect to that for grasping in Experiment 1. The authors interpret their data such that grasping is only affected by visual illusions if grasping is easy (as in Experiment 2 with the posts) and that there is only an illusion effect on manual estimation if vision of the hand is allowed (as in their Experiment 3). Both conclusions, however, contradict the existing literature: The difficulty of grasping in their Experiment 1 was comparable to that in all the other studies (they had used discs of 5-mm thickness, while, for example, Aglioti et al., 1995, Haffenden \& Goodale, 1998, and Haffenden et al., 2001, had used discs of 3-mm thickness). Therefore, if their conclusion 
were correct, all these other studies should have measured larger illusion effects on grasping than on their perceptual measures. This is clearly not the case. The second conclusion is also problematic, because manual estimation is typically performed without vision of the hand. For example, Haffenden and Goodale (1998) and Haffenden et al. (2001) did not allow vision of the hand during manual estimation. If their conclusions were correct, then all these studies should not have measured an effect of the illusion on manual estimation. Again, this is not the case.

We therefore think that this study is inconclusive and needs replication before we can draw strong conclusions from it. Reasons for the difficult-to-interpret results might be that all comparisons were performed between groups (for each task another group of participants was used), that a quite small number of repetitions were used per subjects (from each participant only 32 trials were analysed; Vishton \& Fabre, p. 382), and that given this design the sample sizes were relatively low (for grasping and manual estimation, $N=10 \ldots 16)$. All this might lead to quite variable results in grasping and manual estimation, which are difficult to interpret. Also, note that there is another problem with this study: Vishton and Fabre (2003) calculated the illusion effects in percentages in such a way that the illusion effect in grasping is systematically underestimated. We discuss this problem and possible remedies in "Appendix A: Why do researchers report illusion effects in millimetres instead of percentages?".

The second study on bimanual grasping is by Dewar and Carey (2006). The authors presented the fin-out and fin-in variants of the Müller-Lyer illusion concurrently and had participants either grasp with both hands or perform the manual estimation task. They found illusion effects on manual estimation but not on grasping. This was true even after they calculated corrected illusion effects (as suggested by us, cf. "Classic perception or manual estimation?"). This result would be in line with the perception-versus-action hypothesis.

However, this study has a serious limitation: Grasping is performed under full vision of stimuli and hand. This makes a big difference for the Müller-Lyer illusion: If participants see their hand during grasping, the illusion is largely reduced. This might be due to visual feedback mechanisms. In a recent study (Franz et al., 2007), we tested this in detail: We had participants grasp the Müller-Lyer figure and varied the amount of visual feedback. The illusion effect on grasping strongly depended on the availability of visual feedback: With full vision of the hand during grasping, the motor illusion was very small. If vision was available for one third and two thirds of the grasping movement the illusion increased. If grasping was performed open loop (i.e., vision was available only until movement initiation), then the motor illusion was of the same size as the perceptual illusion. This replicated and extended earlier studies. For example, Westwood et al. (2001) also found a relatively small motor illusion under full-vision conditions, but a much larger in an open-loop condition.

Already Post and Welch (1996) have argued that a dissociation between perception and action can only be convincingly shown under open-loop conditions, because the availability of visual feedback can lead to online corrections (Woodworth, 1899). These online corrections will selectively reduce the measured illusion in grasping, but not in perception. Note, that proponents of the perception-versus-action hypothesis also agree with this analysis (e.g., Haffenden \& Goodale, 1998). Therefore, the study of Dewar and Carey (2006) is not conclusive as evidence for a perceptionversus-action dissociation. The same is true for an earlier study by Otto-de Haart et al. (1999)who used a similar design with unimanual grasping in the Müller-Lyer illusion and found larger illusion effects on perception than on grasping under full-vision conditions.

In short, bimanual grasping poses methodological and conceptual problems. While it might be possible to overcome these problems, the two existing studies on bimanual grasping are inconclusive: Vishton and Fabre (2003) obtained highly unusual results that are very difficult to interpret, and Dewar and Carey (2006) used a full-vision condition that selectively reduces the Müller-Lyer illusion in grasping (probably due to feedback). 


\section{Left-handed versus right-handed grasping}

A recent study (Gonzalez et al., 2006a) compared left-handed grasping with right-handed grasping in the Ebbinghaus and Ponzo illusions. The authors found strong illusion effects if participants used their left hand for grasping, while the effects on right-handed grasping were smaller and in most conditions not significant. This was true independent of whether participants were rightor left-handers. The authors conclude from this that the dorsal vision-for-action system is located in the left hemisphere and that therefore only the right hand has access to the veridical size estimate in the vision-for-action system, while the left hand has to rely on ventral information.

The finding that the left hand is so much more susceptible to the illusions is, of course, quite surprising and interesting. ${ }^{1}$ At the moment we do not have a good explanation for this and therefore are eager to see whether this finding can be replicated. Note, however, that other studies do not give indications for such a dissociation between left and right hand: The bimanual study of Dewar and Carey (2006), which we discussed above, did not find any difference in the illusion effects between left and right hand (they even pooled the data of left and right hand because they were so similar, p. 1505). A study by Radoeva et al. (2005), which also investigated the Müller-Lyer illusion in patients, did also find no difference between left- and right-hand illusion effects in grasping in their healthy control group (only this group performed both types of grasp). So, we tend to wait for further empirical evidence of this effect before drawing strong conclusions from it. Note also that the conclusion, that the dorsal visionfor-action system is located in the left hemisphere (independent of whether the person is left or right-handed), has been criticized as being implausible on other grounds (Derakhshan, 2006; see also the reply by Gonzalez, Goodale, and Ganel, 2006b, in which they state that their proposal is an "admittedly tentative idea", p. 3557). If the proposal is correct then the left hand would not be able to benefit from all the evolutionary advantages of the dorsal vision-foraction system (fast, accurate, metrically accurate) that have been proposed by Milner and Goodale (1995). This serious disadvantage of the left hand would even affect the dominant left hand of left-handers.

\section{Other illusions}

A number of studies on other illusions (Franz et al., 2001; Vishton et al., 1999) and other motor responses (Mon-Williams \& Bull, 2000; Post \& Welch, 1996; van Donkelaar, 1999) also came to the conclusion that perception and action are affected in a similar way. As can be expected, other authors came to different conclusions (e.g., Brenner \& Smeets, 1996; Hu \& Goodale, 2000; Jackson \& Shaw, 2000; Stöttinger \& Perner, 2006; Westwood \& Goodale, 2003), some of which support the perception-versus-action hypothesis and others of which do not and suggest different interpretations. However, all these other illusions were not as systematically investigated as the Ebbinghaus illusion. Therefore, we concentrated in our review on the Ebbinghaus illusion-as the standard example for such a dissociation. We showed in our review that there are some methodological subtleties involved when performing a comparison between perception and action and think that it would be beneficial to transform our arguments also to the evaluation of other studies. For example, studies using manual estimation as a perceptual measure for the illusion typically did not correct for the different slopes of the response functions. Without this correction, however, the comparison between manual estimation and grasping or other dependent measures is not meaningful. Similarly, our argument regarding

\footnotetext{
${ }^{1}$ The other finding-namely that the influences of the illusion on the right hand were not significant in most of the illusion conditions-does not seem very surprising to us, given that for grasping a relatively small number of repetitions were run (each participant performed only 16 illusion grasps with each hand), and that this was not compensated by a large sample size (in each group there were 13 participants).
} 
direct and separate comparisons are relevant for the evaluation of studies on other illusions.

For example, the study of Brenner and Smeets (1996) used the Aglioti paradigm with a direct comparison to investigate the Ponzo illusion. They found a smaller influence of the illusion on MGA in grasping $(0.3 \mathrm{~mm})$ than on perception $(0.8 \mathrm{~mm}$; although they did not test whether this difference is significant, see also "Appendix B: The significant/not-significant-as-difference fallacy”). However, this difference could be due to the same mismatch in task demands that we found for the Ebbinghaus illusion: Brenner and Smeets (1996) measured perception using a direct comparison where participants compared two objects both being subjected to the illusion at the same time, while participants successively grasped always only one or the other of the objects. Therefore, their estimate for the perceptual illusion was probably an overestimate. This could also explain why the influence on grasping was not significant in this study - the sample size of 8 participants was very small to capture an effect on MGA smaller than $0.8 \mathrm{~mm}$. This criticism also applies to the replication of Jackson and Shaw (2000).

Note that this criticism is, however, not a serious problem for the main conclusion of these studies. Their focus was not on a comparison of the illusion effects on perception and MGA (this can be seen by the fact that Jackson and Shaw, 2000, did not even report the size of the perceptual illusion, and Brenner and Smeets, 1996, only reported details of the perceptual task in a footnote). The main focus of these studies was on the question whether grip force was affected by the illusion at all-which it was. Brenner and Smeets (1996) count this as evidence against the perception-versus-action hypothesis because it shows an effect of the illusion on action (and explain the supposed lack of illusion effects on MGA with their grasp model which assumes that MGA does not respond to object size, but to the position of grasp points (see our discussion below). Jackson and Shaw (2000) interpret the illusion effects on grip force as an indication of crosstalk between the vision-for-action and vision-for-perception systems.

\section{The effects of temporal delay on the motor illusion}

It has been reported repeatedly that the effects of visual illusions on motor behaviour increase if a delay is introduced between stimulus presentation and execution of the movement (e.g., Bridgeman, Peery, \& Anand, 1997; Gentilucci, Chieffi, Daprati, Saetti, \& Toni, 1996; Hu \& Goodale, 2000; Westwood \& Goodale, 2003; Westwood et al., 2001). In Milner and Goodale's (1995) framework this is interpreted as an increasing influence of the vision-for-perception system (because the vision-for-action system is assumed to have too short a memory).

However, recently it has been shown that for one classic example, the induced Roelofs effect with pointing movements and saccades (Figure 2e; Bridgeman et al., 1997), this increase can be better explained by a common representation model (Dassonville \& Bala, 2004b; Dassonville, Bridgeman, Bala, Thiem, \& Sampanes, 2004; see also the discussion below in the section "Bridgeman: Cognitive versus sensorimotor maps"). Therefore, it will be an interesting question whether an explanation based on a common representation of object size in perception and action can also be found for reports of increasing illusion effects after a delay in grasping. A full discussion of this topic would go beyond the scope of this article (in fact, it would require another review of similar length as this review). However, we want to sketch some thoughts on this topic here.

For grasping, a first and very prominent study was conducted by $\mathrm{Hu}$ and Goodale (2000). Participants grasped a block, which was accompanied by a second block that was either smaller or larger than the target block. This second block induced a size contrast effect (as measured by manual estimation in their Experiment 3). $\mathrm{Hu}$ and Goodale found in two experiments that the illusion effects on grasping in the no-delay condition (grasping was performed open loop immediately after presentation of the stimuli) were not different from zero, while after a 5-s delay the illusion effects were significantly different from zero. $\mathrm{Hu}$ and Goodale concluded from this pattern of results that the illusion effect 
in grasping increased due to the delay. However, this inference is not correct. To come to this conclusion, they would have to test the difference between the illusion effects in the no-delay and the delay conditions. We calculated this test from their published data and found that the difference was not significant in both experiments $(p=.42$ and $p=.10$ for Experiments 1 and 2, respectively; see "Appendix B: The significant/ not-significant-as-difference fallacy”). In consequence, we see a serious problem for the interpretation of this study and think that it cannot be counted as strong evidence for an increasing illusion effect after a delay.

We recently also started to conduct experiments on this topic (Franz et al., 2007), employing grasping and the Müller-Lyer illusion (which is the "typical" illusion in research on temporal delay; e.g., Gentilucci et al., 1996; Westwood et al., 2001). We were able to replicate the basic effect: The illusion exerted a larger effect on grasping if a delay of $5 \mathrm{~s}$ was introduced as compared to grasping under full vision. However, we showed that this increase was due to the availability of visual feedback: When we successively reduced the amount of visual feedback available during execution of the movement, the motor illusion increased until in the open-loop condition (vision of hand and stimuli was suppressed as soon as the movement had started) the illusion was at the level of the perceptual illusion. Introducing an additional delay of $5 \mathrm{~s}$ did not change the illusion any more, thereby providing evidence against the notions that the illusion does not affect open-loop grasping and that there is a increase of the illusion after a delay due to a shift from dorsal to ventral control of the movement. Interestingly, our findings fit very well to the results of Westwood et al. (2001): They also found an increase of the Müller-Lyer illusion in grasping between their full-vision and open-loop conditions. Introducing an additional delay of up to $2 \mathrm{~s}$ did not change the motor illusion any more.

In short, we did not intend to exhaustively discuss the issue of temporal delay and motor effects of visual illusions here. However, we hope to have shown that the issue of temporal delay is not settled yet. It might well be that further research shows that for the effects of temporal delay on motor illusions similar, simple explanations can be found, as we proposed above for the case of the Müller-Lyer illusion (Franz et al., 2007).

\section{More detail: 4. Alternative theoretical accounts}

In this section we sketch some alternative theoretical accounts (other than the perception-versusaction hypothesis) that have been proposed.

\section{Bridgeman: Cognitive versus sensorimotor maps}

The perception-versus-action distinction of Goodale and Milner (1992; Milner \& Goodale, $1995)$ is similar to Bridgeman's earlier distinction of cognitive versus sensorimotor maps (Bridgeman, Kirch, \& Sperling, 1981; Bridgeman et al., 1997). For a long time, the evidence for this model seemed quite unequivocal: Certain visual illusions that affected the perceived position of an object did not affect pointing movements to these same locations. The strongest findings were obtained with the induced Roelofs effect (Figure 2e; Bridgeman et al., 1997): While perception of the target object is deceived by a surrounding frame that is offset to one side, pointing movements to the same target were accurate. Therefore, Bridgeman suggested that there are two separate spatial maps, one for perception and one for action, thereby also suggesting that perception is prone to the illusion while actions seemed immune.

However, recent evidence by Dassonville, Bridgeman, and coworkers (Dassonville \& Bala, 2004a, 2004b; Dassonville et al., 2004) suggests that these findings are caused by a frame-induced distortion of the observers' apparent midline. They showed that actions guided to targets within the distorted reference frame are accurate because errors of target localization cancel the errors of motor guidance. For perception, on the other side, these errors will not cancel such that the illusion only seems to affect perception and not action. These findings therefore provide a simple explanation for the apparent dissociation between perception and action without having to 
assume separate neural systems for perception and action. Instead, their explanation also assumes a common representation of space, thereby corroborating our results regarding the Ebbinghaus illusion (see also the discussion in Smeets \& Brenner, 2006).

Glover and Dixon: Planning-control model

Glover and Dixon (2002; see also Glover, 2002, 2004) argued that it is possible to find dissociations between perception and action over the time course of grasp movements. They suggested that early parts of a movement are controlled by the ventral cortical stream (and are therefore deceived by contextual illusions) while late parts are controlled by the dorsal stream (and are therefore not deceived by contextual illusions). We (Franz, 2003b, 2004; Franz et al., 2005) replicated one of their studies (Glover \& Dixon, 2002) on the Ebbinghaus illusion and showed that these dynamic illusion effects are likely due to artefacts that were caused by inclusion in the analysis of data points at which the hand already touched the target object. This reduced the illusion effect in late phases of the movements. After exclusion of these contaminated parts of the trajectories we found that the illusion effects were remarkably constant over time.

This is consistent with a number of other studies that also came to similar negative evaluation of Glover and Dixon's (2002) model, using a number of different paradigms (Handlovsky, Hansen, Lee, \& Elliott, 2004; Heath, Rival, \& Binsted, 2004; Heath, Rival, Neely, \& Krigolson, 2006; Heath, Rival, Westwood, \& Neely, 2005; Mendoza, Elliott, Meegan, Lyons, \& Welsh, 2006; Mendoza, Hansen, Glazebrook, Keetch, \& Elliott, 2005). Note that Glover and Dixon's model is also at odds with the perception-versusaction model of Milner and Goodale (Goodale \& Milner, 2004), such that, logically, at least one of the models must be wrong.

\section{Brenner and Smeets: Grasping is not guided by object size}

Some researchers questioned whether the Aglioti paradigm could be used at all as positive evidence for the perception-versus-action hypothesiseven if a clear dissociation between perception and action were found in this paradigm.

Smeets and Brenner (2006) argue that the Aglioti et al. (1995) study and its interpretation in terms of the perception-versus-action hypothesis rest on the assumption that the perceptual measure and MGA in grasping are both measures of object size. Otherwise it would not make sense to compare these measures. Smeets, Brenner, and colleagues raised concerns against this assumption-and therefore questioned whether studies on visual illusion that use the MGA can tell us anything about a potential perception-action dissociation at all. Consequently, they argue that "whether an illusion influences the execution of a task will therefore depend on which spatial attributes are used rather than on whether the task is perceptual or motor" (Smeets, Brenner, Grave, \& Cujpers, 2002; p. 135).

In general, we agree with their main conclusion that the task demands affect which spatial attributes are used. However, we differ with respect to the interpretation of the MGA: We think that even if the basic assumption of Aglioti et al. (1995) is correct-namely that MGA is a measure of object size-the empirical evidence votes against a perception-action dissociation.

Let us sketch why Smeets, Brenner, and colleagues argue against the use of MGA as a measure of object size: Smeets et al. (2002) argue that attributes of objects, such as colour, shape, size, or orientation are often physically related, but do not need to be always perceived consistently. For example, the perceived orientation of a bar might be inconsistent with the perceived location of its end-points. A classic example is the Müller-Lyer illusion in which the fins change the perceived length of the shaft, but not the perceived position of the end-points of the shaft (Gillam \& Chambers, 1985; Mack, Heuer, Villardi, \& Chambers, 1985). In addition, Smeets and Brenner (1999) proposed a new model for grasping that is different from earlier models (e.g., Jeannerod, 1981) and assumes that in grasping we move index finger and thumb independently to different positions. In consequence, 
they interpret the fact that MGA depends linearly on object size (Jeannerod, 1986) as not indicating that MGA is determined by object size, but by the position of the grasp points. Now, if we assume that the Ebbinghaus illusion changes only the size estimate of the object (but not the positions of the grasp points), then it would be no surprise if grasping were less affected by this illusion than was perception.

Smeets, Brenner, and colleagues performed a number of studies to test this notion. For example, Brenner and Smeets (1996) measured lift force in the Ponzo illusion as a dependent measure that might be better suited to measure size information in grasping than MGA-and indeed found illusion effects on lift force. In consequence, they conclude that Aglioti et al. (1995) simply used with MGA the wrong measure. Had they used a motor measure that takes into account the size of the object appropriately, they would have found illusion effects on the motor system (see also the discussion of this study above).

In another study, de Grave, Biegstraaten, Smeets, and Brenner (2005) used the Ebbinghaus illusion with four context elements and rotated the context elements by 45 degrees. For both orientations of the context elements they found similar, significant effects of the illusion on MGA. In addition they found a small but significant effect on the orientation of the hand: The hand orientation at MGA changed by approximately 1 degree in response to the 45-degree rotation of the context elements. They conclude that the Ebbinghaus illusion can influence grasping parameters that are not related to size perception. Since the MGA is not necessarily independent of such influences, the magnitude of the illusory effects on perception and MGA need not be identical - and we should be cautious in attributing apparent differences (or similarities) between perception and grasping to a perception-action dissociation (p. 64).
However, we are not convinced by the argument of de Grave et al. (2005), because it is well known that MGA depends not only on object size alone. For example, if we changed the speed of the movement or the distance between grasp object and participant or a lot of other parameters, all this will affect MGA. Therefore it seems not very surprising that rotating the context elements has some effect on the grasp trajectory. (Note, however, that rotating the context elements did not even affect the MGA; cf. Figure $5 \mathrm{c}$ of de Grave et al., 2005. There was only a small effect on the orientation of the hand at the time of MGA.) The critical question is what happens if we keep all these other parameters constant, whether then MGA responds to object size. And this seems to be the case.

Another, recent study (Gilster, KuhtzBuschbeck, Wiesner, \& Ferstl, 2006) came to a similar view as Brenner, Smeets, and colleagues on the appropriateness of comparisons between the effects of the Ebbinghaus illusion on perception and on MGA in grasping. Gilster et al. (2006) investigated the Ebbinghaus illusion in grasping in a similar way as the example studies, but added a neutral condition without context elements. Again, they found effects of the illusion on grasping: MGA was larger with small context elements than with large context elements. However, in the neutral condition MGA was larger than in the condition with small context elements. Gilster et al. (2006) concluded from this that the presence of the context elements leads to a general reduction in MGA and that this "reduction-effect" might also reduce potential effects of visual illusions on grasping. Therefore, they argue that larger effects of the Ebbinghaus illusion on perception than on grasping cannot be unambiguously interpreted in favour of the perception-versus-action hypothesis. ${ }^{2}$

\footnotetext{
${ }^{2}$ One might be tempted to interpret the results of Gilster et al. (2006) as evidence for the large-gap-small-grasp mechanism (Haffenden \& Goodale, 2000; Haffenden et al., 2001). However, Haffenden and Goodale assume that participants grasp relatively large in the small-near figure because they somehow embrace the entire Ebbinghaus figure. But if that is the case, then they should grasp smaller in the neutral condition because the context elements are removed. This is the opposite of the reduction effect reported by Gilster et al. (2006).
} 
We think, however, that this conclusion might be premature. Even in perception the neutral condition without context circles can lead to a larger perceived size than does the condition with small context circles (e.g., Girgus et al., 1972; Roberts et al., 2005). Unfortunately, a quantitative comparison between perceptual and motor effects of the illusion is missing in the study of Gilster et al. (2006), such that we do not know whether there are really important differences between the perceptual and motor effects of the illusion in this study. Also, Pavani et al. (1999) used another neutral condition in which context elements of equal size as the central element were used and showed that relative to this neutral condition, the effects of the illusion on perception and grasping were again very similar.

In short, we think the notion that we have to consider carefully which spatial attributes are used during the perceptual or motor tasks is very important and worthwhile. Smeets, Brenner, and colleagues use this idea together with their specific grasp model to argue that even if certain visual illusion affected MGA less than perception this would not constitute evidence for the perception-action model. We took a different research strategy: We first wanted to clarify whether certain visual illusions (such as the Ebbinghaus illusion) do indeed affect MGA less than perception. And, in our view, this does not seem to be the case.

\section{GENERAL DISCUSSION}

We argued that the grasp data on the Ebbinghaus illusion are surprisingly consistent. The perceptual data are not as consistent. However, if we use such standard precautions as taking into account the slope of the measures' response functions and making sure that all tasks are appropriately matched, then we can explain the differences between classic perceptual measures, manual estimation, and grasping. In consequence, we find that all these measures are similarly affected by the Ebbinghaus illusion.

This leads to a simple, parsimonious interpretation of the data: The Ebbinghaus illusion deceives a common representation of object size. This representation is used by perception and by the action system. For us, as scientists, this should be good news: We have consistent data, and we have a simple, parsimonious interpretation.

However, this conclusion is at odds with the notion that grasping visual illusions provides strong, positive evidence for the perceptionversus-action hypothesis of Goodale and Milner (1992; Milner \& Goodale, 1995). Let us first illustrate that these findings have indeed been counted as strong, positive evidence for the theory: For example, Haffenden and Goodale (1998) wrote with respect to their findings on the Ebbinghaus illusion: "The results of the present experiment provide strong support for the idea that the visual mechanisms underlying perception are distinct from those underlying the control of skilled actions" (p. 129). Similarly, Plodowski and Jackson (2001) judged with respect to the Haffenden et al. (2001) study on the Ebbinghaus illusion: "Goodale and colleagues have obtained compelling new evidence in support of their proposed dichotomy between visual processing for perception and motor control" (p. R304). Clearly, if strong, compelling evidence for a theory turns out to be problematic, then our believe in this theory should somehow be affected.

We are not claiming that our findings necessarily disprove the perception-versus-action hypothesis. In fact, we could easily reconcile the perception-versus-action hypothesis with our findings in three simple ways (cf. Franz et al., 2001): (a) The Ebbinghaus illusion could be created in each of the systems redundantly-and happen to be of exactly the same size in the two systems. We call this the "redundant illusion hypothesis" in the following. (b) We could assume that there is strong crosstalk between the two systems, which contaminates the veridical size estimate in the action system so strongly that grasping is as much deceived by the illusion as is perception. We call this the "strong crosstalk hypothesis". (c) We could assume that the Ebbinghaus illusion is created relatively early, before the dorsal and ventral streams separate. We call this the "early illusion hypothesis". 
How plausible are these hypotheses? The redundant illusion hypothesis and the strong crosstalk hypothesis both are scientifically problematic because they simply increase the number of entities and are therefore not parsimonious. As long as we do not have other, independent evidence that indicates that such entities exist (i.e., that the illusion is created twice in parallel, or that the action system calculates the veridical size but that this is then overwritten by crosstalk from the perceptual system), these hypotheses are simply post hoc explanations, and we do not see much value in them. Note, however, that this does not mean that these hypotheses are necessarily wrong or worthless. It could well be that some new findings indicate that indeed one of these two hypotheses is close to the truth.

The early illusion hypothesis, on the other side, could be an interesting possibility, and it is quite surprising that the proponents of the perceptionversus-action hypothesis seem to be opposing this possibility. Milner and Dyde (2003) discuss this possibility for other visual illusions, but dismiss it for the Ebbinghaus illusion. Our discussion above on the mechanisms of the Ebbinghaus illusion (see: "More detail: 2. How is the Ebbinghaus illusion generated?") shows that currently not much is know on the exact sources of the Ebbinghaus illusion. Clarifying this question will be a task for future research.

However, all three hypotheses can only reconcile the perception-versus-action hypothesis with our finding of similar illusion effects for perception and action. If we accept one of these hypotheses, then grasping visual illusions cannot provide strong, positive evidence for the perception-versus-action hypothesis any more. In consequence, it is also possible that the perception-versus-action hypothesis is simply wrong and should be rejected. This will depend on the strength of the other, independent evidence that has been put forward in support of the perception-versus-action hypothesis. A full evaluation would go far beyond the scope of this article. Note, however, that other evidence that has traditionally been counted as strongly supporting the perception-versus-action hypothesis also turned out to be problematic. For example, the classic distinction of cognitive versus sensorimotor maps (Bridgeman et al., 1981, 1997) has been challenged (see our discussion above). Similarly, Schenk (2006) questioned whether the dissociation in the famous patient D.F. is really between perception and action as suggested by Goodale and Milner (1992) or maybe between different task demands. Other researchers raised further concerns against Goodale and Milner's interpretation of the patient data (e.g., Pisella, Binkofski, Lasek, Toni, \& Rossetti, 2006). Taken together, all this criticism might lead us to the conclusion that the brain is not subdivided in two parallel systems, which perform certain calculations twice. Specifically, the notion that object size is calculated twice, once in the ventral stream for perception (deceived by visual illusions and conscious) and once in the dorsal stream for action (veridical and unconscious), loses much of its strength if one follows our arguments on visual illusions and grasping.

First published online 13 June 2008

\section{REFERENCES}

Aglioti, S., DeSouza, J. F. X., \& Goodale, M. A. (1995). Size-contrast illusions deceive the eye but not the hand. Current Biology, 5, 679-685.

Bartelt, R., \& Darling, W. G. (2002). Opposite effects on perception and action induced by the Ponzo illusion. Experimental Brain Research, 146, 433-440.

Brenner, E., \& Smeets, J. B. J. (1996). Size illusion influences how we lift but not how we grasp an object. Experimental Brain Research, 111, 473-476.

Bridgeman, B., Kirch, M., \& Sperling, A. (1981). Segregation of cognitive and motor aspects of visual function using induced motion. Perception $\varepsilon^{\circ}$ Psychophysics, 29, 336-342.

Bridgeman, B., Peery, S., \& Anand, S. (1997). Interaction of cognitive and sensorimotor maps of visual space. Perception E Psychophysics, 59, 456-469.

Bruno, N. (2001). When does action resist visual illusions? Trends in Cognitive Sciences, 5, 379-382.

\section{COGNITIVE NEUROPSYCHOLOGY, 2008, 25 (7-8)}


Buonaccorsi, J. P. (2001). Fieller's theorem. In A. H. El-Shaarawi \& W. W. Piegorsch (Eds.), Encyclopedia of environmetrics (pp. 773-775). New York: Wiley.

Cantor, G. N. (1956). A note on a methodological error commonly committed in medical and psychological research. American Journal of Mental Deficiency, 61, 17-18.

Carey, D. P. (2001). Do action systems resist visual illusions? Trends in Cognitive Sciences, 5, 109-113.

Choplin, J. M., \& Medin, D. L. (1999). Similarity of the perimeters in the Ebbinghaus illusion. Perception $\mathcal{E}^{\circ}$ Psychophysics, 61, 3-12.

Coren, S., \& Enns, J. T. (1993). Size contrast as a function of conceptual similarity between test and inducers. Perception E Psychophysics, 54, 579-588.

Coren, S., \& Girgus, J. S. (1972). A comparison of five methods of illusion measurement. Behavior Research Methods \& Instrumentation, 4, 240-244.

Coren, S., \& Girgus, J. S. (1973). Visual spatial illusions-many explanations. Science, 179, 503-504.

Coren, S., \& Miller, J. (1974). Size contrast as a function of figural similarity. Perception E Psychophysics, 16, 355-357.

Daprati, E., \& Gentilucci, M. (1997). Grasping an illusion. Neuropsychologia, 35, 1577-1582.

Dassonville, P., \& Bala, J. K. (2004a). Are the original Roelofs effect and the induced Roelofs effect confounded by the same expansion of remembered space? Vision Research, 44, 1025-1029.

Dassonville, P., \& Bala, J. K. (2004b). Perception, action, \& Roelofs effect: A mere illusion of dissociation. Public Library of Science/Biology, 2, 1936-1945.

Dassonville, P., Bridgeman, B., Bala, J. K., Thiem, P., \& Sampanes, A. (2004). The induced Roelofs effect: Two visual systems or the shift of a single reference frame? Vision Research, 44, 603-611.

Day, R. H. (1972). Visual spatial illusions: A general explanation. Science, 175, 1335-1340.

de Grave, D. D. J., Biegstraaten, M., Smeets, J. B. J., \& Brenner, E. (2005). Effects of the Ebbinghaus figure on grasping are not only due to misjudged size. Experimental Brain Research, 163, 58-64.

Delboeuf, J. (1865). Note sur certaines illusions d'optique: Essai d'une théorie psychophysique de la manière dont I'oeil apprécie les distances et les angles [Note on certain optical illusions: Essay on a psychophysical theory concerning the way in which the eye evaluates distances and angles]. Bulletins de l'Académie Royale des Sciences, Lettres et Beaux-arts de Belgique, 19, 195-216.
Derakhshan, I. (2006). Laterality of the command center in relation to handedness and simple reaction time: $\mathrm{A}$ clinical perspective. Journal of Neurophysiology, 96, 3556-3556.

Dewar, M. T., \& Carey, D. P. (2006). Visuomotor "immunity" to perceptual illusion: A mismatch of attentional demands cannot explain the perceptionaction dissociation. Neuropsychologia, 44, 1501-1508.

Dohle, C., Ostermann, G., Hefter, H., \& Freund, H. J. (2000). Different coupling for the reach and grasp components in bimanual prehension movements. Neuroreport, 11, 3787-3791.

Eagleman, D. M. (2001). Visual illusions and neurobiology. Nature Reviews Neuroscience, 2, 920-926.

Ebbinghaus, H. (1902). Grundzüge der Psychologie. [Foundations of psychology] Leipzig, Germany: Viet.

Emmert, E. (1881). Größenverhältnisse der Nachbilder. Klinische Monatsblätter für Augenheilkunde [sizerelations in after images], 19, 443-454.

Fick, A. (1851). De errone quodam optic asymmetria bulbi effecto [About optical errors due to asymmetry]. Marburg: Koch.

Franz, V. H. (2001). Action does not resist visual illusions. Trends in Cognitive Sciences, 5, 457-459.

Franz, V. H. (2003a). Manual size estimation: A neuropsychological measure of perception? Experimental Brain Research, 151, 471-477.

Franz, V. H. (2003b). Planning versus online control: Dynamic illusion effects in grasping? Spatial Vision, 16, 211-223.

Franz, V. H. (2004). Is there a dynamic illusion effect in the motor system? Behavioral and Brain Sciences, 27, 34-35.

Franz, V. H. (2007). Ratios: A short guide to confidence limits and proper use. Manuscript submitted for publication. http://arxiv.org/abs/0710.2024

Franz, V. H., Bülthoff, H. H., \& Fahle, M. (2003). Grasp effects of the Ebbinghaus illusion: Obstacleavoidance is not the explanation. Experimental Brain Research, 149, 470-477.

Franz, V. H., Fahle, M., Bülthoff, H. H., \& Gegenfurtner, K. R. (2001). Effects of visual illusions on grasping. Journal of Experimental Psychology: Human Perception and Performance, 27, 1124-1144.

Franz, V. H., Gegenfurtner, K. R., Bülthoff, H. H., \& Fahle, M. (2000). Grasping visual illusions: No evidence for a dissociation between perception and action. Psychological Science, 11, 20-25.

Franz, V. H., Hesse, C., \& Kollath, S. (2007). Grasping after a delay: More ventral than dorsal? Poster presented at the Vision Sciences Society conference (VSS), Sarasota, Florida. Journal of Vision, 7, 157a. 
Franz, V. H., Scharnowski, F., \& Gegenfurtner, K. R. (2005). Illusion effects on grasping are temporally constant, not dynamic. Journal of Experimental Psychology: Human Perception and Performance, 31, 1359-1378.

Gentilucci, M., Chieffi, S., Daprati, E., Saetti, M. C., \& Toni, I. (1996). Visual illusion and action. Neuropsychologia, 34, 369-376.

Gillam, B., \& Chambers, D. (1985). Size and position are incongruous-measurements on the Müller-Lyer figure. Perception E Psychophysics, 37, 549-556.

Gilster, R., Kuhtz-Buschbeck, J. P., Wiesner, C. D., \& Ferstl, R. (2006). Grasp effects of the Ebbinghaus illusion are ambiguous. Experimental Brain Research, 171, 416-420.

Girgus, J. S., Coren, S., \& Agdern, M. V. R. A. (1972). The interrelationship between the Ebbinghaus and Delboeuf illusions. Journal of Experimental Psychology, 95, 453-455.

Glover, S. (2002). Visual illusions affect planning but not control. Trends in Cognitive Sciences, 6, 288-292.

Glover, S. (2004). Separate visual representations in the planning and control of action. Behavioral and Brain Sciences, 27, 3-23.

Glover, S., \& Dixon, P. (2002). Dynamic effects of the Ebbinghaus illusion in grasping: Support for a planning/control model of action. Perception $\mathcal{E}^{\circ}$ Psychophysics, 64, 266-278.

Gonzalez, C. L. R., Ganel, T., \& Goodale, M. A. (2006a). Hemispheric specialization for the visual control of action is independent of handedness. Journal of Neurophysiology, 95, 3496-3501.

Gonzalez, C. L. R., Goodale, M. A., \& Ganel, T. (2006b). Laterality of the command center in relation to handedness and simple reaction time: A clinical perspective-reply. Journal of Neurophysiology, 96, 3557.

Goodale, M. A. (2006, October). Visual duplicity: Action without perception in the human visual system. The XIV. Kanizsa lecture, Trieste, Italy.

Goodale, M. A., Kroliczak, G., \& Westwood, D. A. (2005). Dual routes to action: Contributions of the dorsal and ventral streams to adaptive behavior. Progress in Brain Research, 149, 269-283.

Goodale, M. A., Meenan, J. P., Bülthoff, H. H., Nicolle, D. A., Murphy, K. J., \& Carolynn, I. R. (1994). Separate neural pathways for the visual analysis of object shape in perception and prehension. Current Biology, 4, 604-610.

Goodale, M. A., \& Milner, A. D. (1992). Separate visual pathways for perception and action. Trends in Neurosciences, 15, 97-112.
Goodale, M. A., \& Milner, A. D. (2004). Plans for action. Behavioral and Brain Sciences, 27, 37-40.

Goodale, M. A., Milner, A. D., Jakobson, L. S., \& Carey, D. P. (1991). A neurological dissociation between perceiving objects and grasping them. Nature, 349, 154-156.

Goodale, M. A., \& Westwood, D. A. (2004). An evolving view of duplex vision: Separate but interacting cortical pathways for perception and action. Current Opinion in Neurobiology, 14, 203-211.

Gregory, R. L. (1963). Distortion of visual space as inappropriate constancy scaling. Nature, 199, 678-680.

Haffenden, A. M., \& Goodale, M. A. (1998). The effect of pictorial illusion on prehension and perception. Journal of Cognitive Neuroscience, 10, 122-136.

Haffenden, A. M., \& Goodale, M. A. (2000). Independent effects of pictorial displays on perception and action. Vision Research, 40, 1597-1607.

Haffenden, A. M., Schiff, K. C., \& Goodale, M. A. (2001). The dissociation between perception and action in the Ebbinghaus illusion: Nonillusory effects of pictorial cues on grasp. Current Biology, 11, 177-181.

Haffenden, A. M., Zavitz, K. J., \& Goodale, M. A. (2000). 2-D stimuli adjacent to contact points on 3-D targets for grasp are treated as obstacles by the visuomotor system. Investigative Ophthalmology $\mathcal{E}^{\circ}$ Visual Science, 41, S812-S812.

Handlovsky, I., Hansen, S., Lee, T. D., \& Elliott, D. (2004). The Ebbinghaus illusion affects on-line movement control. Neuroscience Letters, 366, 308-311.

Heath, M., Rival, C., \& Binsted, G. (2004). Can the motor system resolve a premovement bias in grip aperture? Online analysis of grasping the MüllerLyer illusion. Experimental Brain Research, 158, 378-384.

Heath, M., Rival, C., Neely, K., \& Krigolson, O. (2006). Müller-Lyer figures influence the online reorganization of visually guided grasping movements. Experimental Brain Research, 169, 473-481.

Heath, M., Rival, C., Westwood, D. A., \& Neely, K. (2005). Time course analysis of closed- and openloop grasping of the Müller-Lyer illusion. Journal of Motor Behavior, 37, 179-185.

Holway, A. H., \& Boring, E. G. (1941). Determinants of apparent visual size with distance variant. American Journal of Psychology, 54, 21-37.

$\mathrm{Hu}$, Y., \& Goodale, M. A. (2000). Grasping after a delay shifts size-scaling from absolute to relative metrics. Journal of Cognitive Neuroscience, 12, 856-868.

\section{6}

COGNITIVE NEUROPSYCHOLOGY, 2008, 25 (7-8) 
Jackson, G. M., German, K., \& Peacock, K. (2002). Functional coupling between the limbs during bimanual reach-to-grasp movements. Human Movement Science, 21, 317-333.

Jackson, G. M., Jackson, S. R., \& Kritikos, A. (1999). Attention for action: Coordinating bimanual reachto-grasp movements. British Journal of Psychology, 90, 247-270.

Jackson, S. R., \& Shaw, A. (2000). The Ponzo illusion affects grip-force but not grip-aperture scaling during prehension movements. Journal of Experimental Psychology: Human Perception and Performance, 26, 418-423.

Jacob, P., \& Jeannerod, M. (1999). Consciousness and the visuomotor transformation. Retrieved February 19, 2008 from the CNRS Institute of Cognitive Sciences Web site: http://www.isc.cnrs.fr/wp/wp99-13.htm

Jaeger, T. (1978). Ebbinghaus illusions: Size contrast or contour interaction phenomena? Perception $\mathcal{E}^{\circ}$ Psychophysics, 24, 337-342.

Jaeger, T., \& Pollack, R. H. (1977). Effect of contrast level and temporal order on the Ebbinghaus circles illusion. Perception E Psychophysics, 21, 83-87.

Jeannerod, M. (1981). Intersegmental coordination during reaching at natural visual objects. In J. Long \& A. Baddeley (Eds.), Attention and performance (Vol. 9, pp. 153-168). Hillsdale, NJ: Lawrence Erlbaum Associates, Inc.

Jeannerod, M. (1984). The timing of natural prehension movements. Journal of Motor Behavior, 16, 235-254.

Jeannerod, M. (1986). The formation of finger grip during prehension: A cortically mediated visuomotor pattern. Behavioral Brain Research, 19, 99-116.

Jeannerod, M., \& Decety, J. (1990). The accuracy of visuomotor transformation. An investigation into the mechanisms of visual recognition of objects. In M. Goodale (Ed.), Vision and action. The control of grasping (pp. 33-45). Norwood, NJ: Ablex.

Mack, A., Heuer, F., Villardi, K., \& Chambers, D. (1985). The dissociation of position and extent in Müller-Lyer figures. Perception E' Psychophysics, 37, 335-344.

Massaro, D. W., \& Anderson, N. H. (1971). Judgemental model of the Ebbinghaus illusion. Journal of Experimental Psychology, 89, 147-151.

Maxwell, S. E. (2004). The persistence of underpowered studies in psychological research: Causes, consequences, \& remedies. Psychological Methods, 9, 147-163.

McCready, D. (1985). On size, distance, \& visual angle perception. Perception E Psychophysics, 37, 323-334.

Mendoza, J., Elliott, D., Meegan, D. V., Lyons, J. L., \& Welsh, T. N. (2006). The effect of the Müller-Lyer illusion on the planning and control of manual aiming movements. Journal of Experimental Psychology-Human Perception and Performance, 32, 413-422.

Mendoza, J., Hansen, S., Glazebrook, C. M., Keetch, K. M., \& Elliott, D. (2005). Visual illusions affect both movement planning and on-line control: A multiple cue position on bias and goaldirected action. Human Movement Science, 24, 760-773.

Milner, A. D., \& Goodale, M. A. (1995). The visual brain in action. Oxford, UK: Oxford University Press.

Milner, A. D., \& Dyde, R. (2003). Why do some perceptual illusions affect visually guided action, when others don't? Trends in Cognitive Sciences, 7, $10-11$.

Mon-Williams, M., \& Bull, R. (2000). The Judd illusion: Evidence for two visual streams or two experimental conditions? Experimental Brain Research, 130, 273-276.

Mon-Williams, M., Tresilian, J. R., Coppard, V. L., \& Carson, R. G. (2001). The effect of obstacle position on reach-to-grasp movements. Experimental Brain Research, 137, 497-501.

Müller-Lyer, F. C. (1889). Optische Urteilstäuschungen [Visual illusions]. Dubois-Reymonds Archive fur Anatomie und Physiologie, Supplement Volume, 263-270.

Murray, S. O., Boyaci, H., \& Kersten, D. (2006). The representation of perceived angular size in human primary visual cortex. Nature Neuroscience, 9, 429-434.

Nicolas, S. (1995). Joseph Delboeuf on visual illusions: A historical sketch. The American Journal of Psychology, 108, 563-574.

Otto-de Haart, G. E., Carey, D. P., \& Milne, A. B. (1999). More thoughts on perceiving and grasping the MüllerLyer illusion. Neuropsychologia, 37, 1437-1444.

Pavani, F., Boscagli, I., Benvenuti, F., Rabuffetti, M., \& Farnè, A. (1999). Are perception and action affected differently by the Titchener circles illusion? Experimental Brain Research, 127, 95-101.

Pavlova, M., \& Sokolov, A. (2000). Speed perception is affected by the Ebbinghaus-Titchener illusion. Perception, 29, 1203-1208.

Pisella, L., Binkofski, F., Lasek, K., Toni, I., \& Rossetti, Y. (2006). No double-dissociation between optic ataxia and visual agnosia: Multiple sub-streams for multiple visuo-manual integrations. Neuropsychologia, 44, 2734-2748.

Plodowski, A., \& Jackson, S. R. (2001). Vision: Getting to grips with the Ebbinghaus illusion. Current Biology, 11, R306-R308. 
Post, R. B., \&Welch, R. B. (1996). Is there dissociation of perceptual and motor responses to figural illusions? Perception, 25, 569-581.

Pressey, A. W. (1977). Measuring the Titchener circles and Delboeuf illusions with the method of adjustment. Bulletin of the Psychonomic Society, 10, 118-120.

Radoeva, P. D., Cohen, J. D., Corballis, P. M., Lukovits, T. G., \& Koleva, S. G. (2005). Hemispheric asymmetry in a dissociation between the visuomotor and visuoperceptual streams. Neuropsychologia, 43, 1763-1773.

Restle, F., \& Merryman, C. T. (1968). An adaptationlevel theory account of a relative-size illusion. Psychonomic Science, 12, 229-230.

Roberts, B., Harris, M. G., \& Yates, T. A. (2005). The roles of inducer size and distance in the Ebbinghaus illusion (Titchener circles). Perception, 34, 847-856.

Roelofs, C. (1935). Optische Lokalisation [Visual localization]. Archiv für Augenheilkunde, 109, 395-415.

Schenk, T. (2006). An allocentric rather than perceptual deficit in patient DF. Nature Neuroscience, 9, 13691370.

Schindler, I., Rice, N. J., McIntosh, R. D., Rossetti, Y., Vighetto, A., \& Milner, A. D. (2004). Automatic avoidance of obstacles is a dorsal stream function: Evidence from optic ataxia. Nature Neuroscience, 7, 779-784.

Smeets, J. B. J., \& Brenner, E. (1999). A new view on grasping. Motor Control, 3, 237-271.

Smeets, J. B. J., \& Brenner, E. (2001). Action beyond our grasp. Trends in Cognitive Sciences, 5, 287.

Smeets, J. B. J., \& Brenner, E. (2006). 10 years of illusions. Journal of Experimental Psychology-Human Perception and Performance, 32, 1501-1504.

Smeets, J. B. J., Brenner, E., Grave, D. D. J. de, \& Cujpers, R. H. (2002). Illusions in action: Consequences of inconsistent processing of spatial attributes. Experimental Brain Research, 147, 135-144.

Stöttinger, E., \& Perner, J. (2006). Dissociating size representation for action and for conscious judgment: Grasping visual illusions without apparent obstacles. Consciousness and Cognition, 15, 269-284.

Thiéry, A. (1896). Über geometrisch-optische Täuschungen [About geometric-optical illusions]. Philosophische Studien, 12, 67-126.

Titchener, E. B. (1901). Experimental psychology: A manual of laboratory practice. New York: MacMillan.

Tresilian, J. R. (1998). Attention in action or obstruction of movement? A kinematic analysis of avoidance behavior in prehension. Experimental Brain Research, 120, 352-368.

van Donkelaar, P. (1999). Pointing movements are affected by size-contrast illusions. Experimental Brain Research, 125, 517-520.

Vishton, P. M., \& Fabre, E. (2003). Effects of the Ebbinghaus illusion on different behaviors: Oneand two-handed grasping; one- and two-handed manual estimation; metric and comparative judgment. Spatial Vision, 16, 377-392.

Vishton, P. M., Pea, J. G., Cutting, J. E., \& Nunez, L. N. (1999). Comparing effects of the horizontalvertical illusion on grip scaling and judgment: Relative versus absolute, not perception versus action. Journal of Experimental Psychology-Human Perception and Performance, 25, 1659-1672.

Wade, N. (1998). Introns-illusions as neuro-signs. Current Biology, 8, R593-R593.

Weintraub, D. J. (1979). Ebbinghaus illusion-context, contour, \& age influence the judged size of a circle amidst circles. Journal of Experimental PsychologyHuman Perception and Performance, 5, 353-364.

Weintraub, D. J., \& Schneck, M. K. (1986). Fragments of Delboeuf and Ebbinghaus illusions-contour context explorations of misjudged circle size. Perception E Psychophysics, 40, 147-158.

Westwood, D. A., Chapman, C. D., \& Roy, E. A. (2000a). Pantomimed actions may be controlled by the ventral visual stream. Experimental Brain Research, 130, 545-548.

Westwood, D. A., Dubrowski, A., Carnahan, H., \& Roy, E. A. (2000b). The effect of illusory size on force production when grasping objects. Experimental Brain Research, 135, 535-543.

Westwood, D. A., \& Goodale, M. A. (2003). Perceptual illusion and the real-time control of action. Spatial Vision, 16, 243-254.

Westwood, D. A., Heath, M., \& Roy, E. A. (2000c). The effect of a pictorial illusion on closed-loop and open-loop prehension. Experimental Brain Research, 134, 456-463.

Westwood, D. A., McEachern, T., \& Roy, E. A. (2001). Delayed grasping of a Müller-Lyer figure. Experimental Brain Research, 141, 166-173.

Woodworth, R. (1899). The accuracy of voluntary movement. Psychological Review Monograph, 3, 1-114.

Wundt, W. (1898). Die geometrisch-optischen Täuschungen [The geometric-optical illusions]. Akademie der sächsischen Wissenschaften Leipzig, Abhandlungen, 24, 53-178. 


\section{APPENDIX A}

\section{Why do researchers report illusion effects in millimetres instead of percentages?}

In perceptual research, illusion effects are sometimes reported in percentages. If, for example, a disc of a physical diameter of $30 \mathrm{~mm}$ is treated as if it had a diameter of $33 \mathrm{~mm}$, then we could describe this as a $[(33-30) / 30] \times 100=10 \%$ illusion effect. This can be useful because we can expect that if we scaled up the whole stimulus configuration such that the diameter of the disc were $40 \mathrm{~mm}$, then there should still be a $10 \%$ illusion effect, and the disc should be treated as if it had a diameter of $44 \mathrm{~mm}$. Researchers on the effects of visual illusions on grasping did not, however, choose this option but preferred to work with absolute sizes - as did many researchers in perceptual research. One reason for this in perceptual research is that the illusion effect can depend on additional factors that might be obscured if we worked in percentages (e.g., Restle \& Merryman, 1968, p. 230). Therefore, we also report illusion effects in millimetres in this review. If we estimate the percentages for the illusion effects shown in Figure 1 for grasping and classic perceptual measures, then they are roughly between $4 \%$ and $5 \%$. However, presenting all the data in percentages would be difficult given that these values and the corresponding standard errors are often not reported in sufficient detail. This does not, however, pose any problems for our comparison because the stimuli were similar enough in the example studies such that there is no necessity to correct for scaling.

One important reason why researchers worked in absolute sizes lies in the fact that the MGA is more complicated than classic perceptual measures: It depends linearly on object size, but with a nonzero intercept and a slope that sometimes deviates from unity. To illustrate the problem, consider the case that for MGA the intercept is $20 \mathrm{~mm}$, and the slope is 0.8 . When grasping a disc of $30-\mathrm{mm}$ diameter, this will result in a MGA of: $20 \mathrm{~mm}+(0.8 \times 30) \mathrm{mm}=44 \mathrm{~mm}$. Now, assume we introduce a $10 \%$ illusion, such that the disc is treated as if it were $33 \mathrm{~mm}$ in diameter. This will result in a MGA of: $20 \mathrm{~mm}+(0.8 \times 33) \mathrm{mm}=46.4 \mathrm{~mm}$. If we now calculated a percentage for the illusion effect in the same way as for classic perceptual measures, we would calculate: [(46.4 - 44)/ 44] $\times 100=5.5 \%$. This would be erroneous (because we constructed our example such that the illusion effect is 10\%). This problem is, for example, present in the study of Vishton and Fabre (2003). The problem arises because the nonzero intercept is not taken adequately into account.

Instead, we have to calculate the physical size that corresponds to a MGA of $46.4 \mathrm{~mm}$ (in our example we know that this is $33 \mathrm{~mm}$, but this would not be known in a real-world situation). For this, we have to invert the linear function and calculate: $(46.4 \mathrm{~mm}-20 \mathrm{~mm}) / 0.8=33 \mathrm{~mm}$. This physical size, corresponding to a MGA of $46.4 \mathrm{~mm}$, can now be used to calculate the percentage in the traditional way: $[(33-30) / 30] \times$ $100=10 \%$. More abstractly, if MGA is related to physical size $(x)$ as: $M G A=\mathrm{a}+\mathrm{b} x$, then we need to calculate the specific physical size $\left(x_{0}\right)$ that corresponds to a specific $\mathrm{MGA}_{0}$ as: $x_{0}=\left(\mathrm{MGA}_{0}-\mathrm{a}\right) / \mathrm{b}$. Only with this value is it correct to calculate a percentage. Note, that this value is closely related to the corrected illusion effects, as are shown in the Figures 1f and 5. For more details and discussions of the corrected illusion effects see Franz (2003a), Franz et al. (2001), and Dewar and Carey (2006).

A final note of caution: In some of these calculations we divide by the slope $b$, which is prone to measurement error. Confidence intervals and statistical tests for such a ratio need special statistical methods. They can be calculated using "Fieller's theorem" or, if b is clearly significantly different from zero, by the delta method (i.e., a linear Taylor approximation). For further details see Buonaccorsi (2001), Franz et al. (2005), and Franz (2007).

\section{APPENDIX B}

\section{The significant/not-significant-as- difference fallacy}

In this appendix we want to describe a statistical inference that was used in quite a few studies on the perception-versus-action topic and which is very problematic. The problem is the following: Quite often, researchers find in one condition a significant illusion effect, while in the other condition the illusion effect is not significantly different from zero. From this pattern of results they conclude that the illusion affected the two conditions differently. However, this is a highly problematic inference. For example, the same pattern of results could be obtained if the illusion affected both conditions to exactly the same degree, but in one condition the variability would be larger, such that only in this condition the illusion effect would not be significantly different from zero.

To come to the conclusion that the two conditions are affected differently by the illusion we would have to test the difference of the illusion effects. Of course, this is a general statistical issue if we compare two or more conditions and is not limited to illusion effects. A similar argument has already been raised earlier by Cantor (1956). ${ }^{3}$

\footnotetext{
${ }^{3}$ There is another issue here, if the comparison is between different measures-for example, between perceptual measure and motor measure. In this case the additional question arises of whether we can compare the perceptual measure and the motor measure at all. We cannot discuss this issue here and refer to the Appendix A of Franz et al. (2001), where we present a mathematical model that shows the assumptions necessary for this comparison.
} 
As an illustrative example, we chose the study of $\mathrm{Hu}$ and Goodale (2000), because this study provides enough statistical detail to be able to demonstrate the problem by recalculation of the data. In their Experiment 1, Hu and Goodale (2000) measured illusion effects on grasping in a no-delay condition and in a 5-s delay condition. The illusion effect in the nodelay condition was not significantly different from zero, while it was significantly different from zero in the delay condition (cf. Figure 4 of $\mathrm{Hu}$ and Goodale, 2000). From this, the authors concluded that the illusion affects the two conditions differently: "The fact that grip scaling becomes sensitive to the relative size of an object after a delay reflects a shift in motor programming from absolute to relative metrics" (p. 861).

However, to come to this conclusion, the authors would have to test the difference of the illusion effects between nodelay and delay conditions. If we perform these calculations (see the next section), we find that the illusion effects were not significantly different $(p=.42)$. The same problem is present in their second experiment, although here the problem is not as pronounced $(p=.10)$. In consequence, the experiments do not provide convincing evidence for the main conclusion of this study-namely, that grasping after a delay is differently affected by the illusion than is immediate grasping.

In summary, not all studies that found differences between perception and action performed the adequate tests. In our main text, this problem is mentioned where appropriate. Ameliorating this problem will have the positive side-effect that larger sample sizes will be used, which might be beneficial for psychology as a cumulative science (Maxwell, 2004).

\section{Tests for the differences in $\mathrm{Hu}$ and Goodale (2000)}

Consider Experiment 1 of $\mathrm{Hu}$ and Goodale (2000): Delay and no-delay conditions were performed in two groups, each with $N$ $=13$ participants. The mean illusion effects were (cf. Figure 4 of $\mathrm{Hu}$ and Goodale, 2000):

$$
\begin{aligned}
& \text { no-delay condition: } \quad I E_{\text {no-delay }}=65.14-64.38=0.76 \\
& \text { delay condition: } \quad I E_{\text {delay }}=68.40-66.61=1.79
\end{aligned}
$$

The illusion effect in the no-delay condition was not significantly different from zero, $F(1,12)=0.535$, $n s$, while it was significantly different from zero in the delay condition, $F(1,12)=$ 6.060, $p<.05$.
Using these data of $\mathrm{Hu}$ and Goodale (2000), we can calculate an independent samples $t$ test for the difference of the illusion effects between the no-delay and delay conditions. For this, we need to calculate the standard error of the mean (SEM) for each condition. This is possible using the two $F$-values given above, which are the results of two separate repeated measures analyses of variance (ANOVAs). For the main effect of the factor illusion these ANOVAs are equivalent to paired $t$ tests. This is so, because: (a) each ANOVA contains only within-subjects factors, (b) the design is balanced, and (c) the factor illusion has only two levels. The $t$ statistics of these $t$ tests calculate as:

$$
\text { no-delay condition: } \begin{aligned}
t(12) & =\sqrt{[F(1,12)]} \\
& =\sqrt{(0.535)=0.731437} \\
\text { delay condition: } t(12) & =\sqrt{[F(1,12)]} \\
& =\sqrt{(6.060)}=2.461707
\end{aligned}
$$

From these $t$ values and from the mean illusion effects, we can calculate the SEMs. This is so because the $t$ statistics are: $t=$ mean $/ S E M$, such that $S E M=$ mean $/ t$. This results in:

no-delay condition:

$$
S E M_{\text {no-delay }}=0.76 / 0.731437=1.039051
$$

delay condition: $\quad S E M_{\text {delay }}=1.79 / 2.461707=0.7271377$

Now we can calculate an independent samples $t$ test on the difference between the no-delay and delay illusion effects. Because the groups have the same sample size, the $t$ statistics calculate as:

$$
\begin{aligned}
t(24) & =\frac{I E_{\text {delay }}-I E_{\text {no-delay }}}{\sqrt{S E M_{\text {delay }}^{2}+S E M_{\text {no-delay }}^{2}}} \\
& =\frac{1.79-0.76}{\sqrt{0.7271377^{2}+1.039051^{2}}}=0.81
\end{aligned}
$$

That is, the difference of the illusion effects in the no-delay and delay conditions of Experiment 1 is not significant, $t(24)=$ $0.81 ; p=.42$. The same calculation for Experiment 2 also results in a not-significant difference, $t(26)=1.68, p=.10$. 